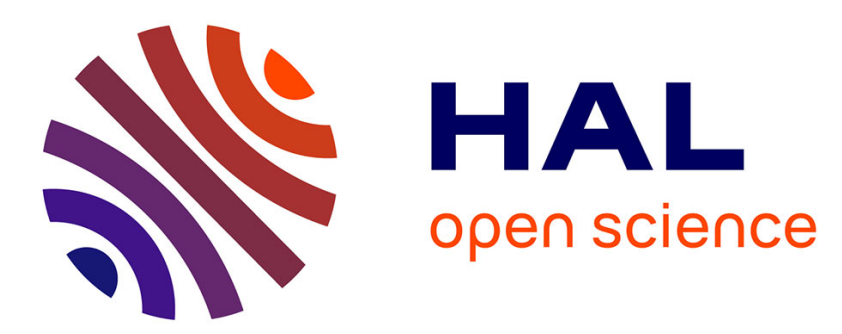

\title{
Le rapport d'Étienne Clémentel (1919). L'avènement administratif des technocrates et de la rationalisation
}

Michel Letté

\section{To cite this version:}

Michel Letté. Le rapport d'Étienne Clémentel (1919). L'avènement administratif des technocrates et de la rationalisation. Documents pour l'histoire des techniques, 2011, 20, pp.167-195. 10.4000/dht.1815. halshs-03224578

\section{HAL Id: halshs-03224578 \\ https://shs.hal.science/halshs-03224578}

Submitted on 11 May 2021

HAL is a multi-disciplinary open access archive for the deposit and dissemination of scientific research documents, whether they are published or not. The documents may come from teaching and research institutions in France or abroad, or from public or private research centers.
L'archive ouverte pluridisciplinaire HAL, est destinée au dépôt et à la diffusion de documents scientifiques de niveau recherche, publiés ou non, émanant des établissements d'enseignement et de recherche français ou étrangers, des laboratoires publics ou privés. 


\title{
Le rapport d'Étienne Clémentel (1919) L'avènement administratif des technocrates et de la rationalisation
}

\author{
Michel Letté \\ CDHTE - Cnam
}

\begin{abstract}
Résumé
Étienne Clémentel, ministre du commerce et de l'industrie de 1915 à 1919, mobilise à partir de 1917 tout ce que compte d'experts auprès de son ministère - c'est-à-dire à la direction des études techniques et au Comité consultatif des arts et manufactures - pour la rédaction d'un monumental bilan de l'industrie française et d'une série de propositions pour la réorganisation économique du pays dans l'après guerre. La présentation dans ce numéro de l'introduction au Rapport général sur lindustrie française, sa situation, son avenir est une invitation à lire ce document selon au moins trois registres d'interprétation et d'analyse. D'abord une lecture guidée par son statut de document administratif composé de données techniques et de recommandations, dont certaines ont vocation à se traduire sous la forme de mesures législatives immédiates. Ensuite la lecture d'un document dont les conditions de sa production invitent à considérer qu'il s' agit surtout d'une entreprise de consolidation des attributions administratives que s'est octroyé le ministère durant la guerre. Ce rapport est en quelque sorte l'acte de naissance d'un véritable ministère de la production industrielle. Enfin, une lecture guidée par la dimension sociale et culturelle d'une proposition de refondre une politique économique et sociale sous l'égide de la rationalisation. En définitive, la production de ce document paraît avoir constitué une sorte de laboratoire de la technocratie politique où leurs partisans auront pu s'exprimer sans complexes durant trois années sur les questions de la réforme sociale par la rationalisation.
\end{abstract}

Résumés et mots clés en anglais sont regroupés en fin de volume, accompagnés des mots clés français

\begin{abstract}
A près une mobilisation sans relâche de plus d'une centaine de techniciens au sein de son ministère. Étienne Clémentel remet le 29 mars 1919 au président du Conseil un volumineux rapport de 2392 pages'. Ce document rassemble statistiques, tableaux, graphiques et données comparées sur l'évolution de la production internationale dans de nombreux

\footnotetext{
1 Ministère du Commerce, de I'Industrie, des Postes et Télégraphes, des Transports maritimes et de la Marine marchande. Direction des études techniques, Rapport général sur l'industrie française, sa situation, son avenir (d'après les travaux des sections du Comité consultatif des arts et manufactures et de la Direction des études techniques). Première partie. Étude de la situation des principales industries avant la guerre et de leur expansion possible (vol. 1 et 2) ; Deuxième partie : Les méthodes d'expansion économique (vol. 3), Paris, Imprimerie nationale, 1919. L'ensemble du rapport Clémentel est en cours de numérisation. II sera prochainement disponible sur le site du Conservatoire numérique (CNUM) du Conservatoire national des arts et métiers (http://cnum.cnam.fr/)
}

secteurs d'activités, avant et pendant la guerre. Il comporte des textes, des bilans, des analyses, 87 rapports d'experts. Ce qui en fait indéniablement une archive de l'économie en guerre. Le chercheur y trouvera des matériaux détaillant des flux de marchandises, ou encore l'état de pénurie pour quelques produits spécifiques. II disposera enfin d'une vue à la fois large et synthétique du paysage commercial et industriel de la France, en tout cas d'une restitution de sa perception par l'administration pendant la guerre. C'est l'usage qu'en a par exemple amplement fait I'historien japonais Jun Sakudo dans ses travaux sur l'histoire de l'industrie chimique française ${ }^{2}$.

À ce titre seul, ce document monumental mérite l'attention. Ce n'est cependant pas la seule justification de son intérêt. D'autres registres

2 Jun Sakudo, Les entreprises de la chimie en France de 1860 à 1932, Bruxelles, Peter Lang, 2011 (trad.), notamment pp. 35-56. 
d'originalité invitent à le lire non seulement comme un matériau pour l'histoire, mais encore comme un objet d'histoire à part entière ${ }^{3}$. Si le rapport d'Étienne Clémentel s'inscrit dans une série de travaux et manifestes supportant l'intervention de l'administration pendant les hostilités, il témoigne surtout de la façon dont les autorités pensent devoir pratiquer l'économie de guerre.

Cette particularité du document est à préciser. II faut saisir le sens de ce qui est écrit, consulter les archives ${ }^{4}$. On y réalisera par exemple que cette entreprise est aussi

3 Ce dont témoignent quelques historiens. II faut ici mentionner les travaux remarquables et pionniers de Clothilde Druelle-Korn, Le Ministère du commerce et de l'industrie pendant la Première guerre mondiale : Étienne Clémentel ou la volonté de moderniser la France, DEA de Paris IEP, 1993 ; Un laboratoire réformateur, le Département du commerce en France et aux États-Unis de la Grande Guerre aux années vingt, Thèse IEP Paris, 2004 et « Ministère du Commerce - Statistique générale de la France. Le rendez-vous manqué des statistiques économiques ", Histoire \& Mesure, 22, 2007, pp. 45-69; Yves Roussel, De la science et de l'industrie: Ministère Clémentel, 1915-1919, DEA de I'EHESS, 1985. Voir aussi ceux de Antoine Prost et Jay Winter, Penser la Grande guerre : un essai d'historiographie, Paris, Sevil, 2004, pp. 145-168; Fabienne Bock, Un parlementarisme de guerre, 1914-1919, Paris, Belin, 2002, 350 p. et «L'exubérance de l'État en France de 1914 à 1918 ", Vingtième Siècle, 3, 1984, pp. 41-52 ; François Caron, "Le rapport Clémentel ", Entreprises et histoire, 3, 1993, pp. 127-129 ; Pierre Rosanvallon, L'État en France, de 1789 à nos jours, Paris, Seuil, 1990, pp. 226-238 ; George-Henri Soutou, L'or et le sang. Les buts de guerre économiques de la Première guerre mondiale, Paris, Fayard, 1989 ; John F. Godfrey, Capitalism at War : Industrial policy and bureaucracy in France, 1914-1918, New York, Berg Publishers Ltd, 1987 ; Adrian Rossiter, Experiments with corporatist politics in republican France, 1916-1939, PHD University of Oxford, 1986 ; Richard F. Kuisel, Le capitalisme et l'État en France. Modernisation et dirigisme au XXe siècle, Paris, Gallimard, 1984, pp. 83-116; Marc Trachtenberg, «The Clementel plan in historical perspective "1, Proceedings of the Annual Meeting of the Western Society for French History, 7, 1979, pp. 142-148 et « "A new Economic Order" : Étienne Clémentel and French Economic Diplomacy during the first world war ", French Historical Studies, 10 (2), 1977, pp. 315-341.

4 Pour l'essentiel, elles concernent la série F/12 des Archives nationales. Voir 7995 : Dossier « après-guerre » : « Rapport à M. le Ministre sur le régime économique de la France » par le capitaine Henry Blazeix, 20 février 1917, 51 p. ; 8029 à 8044 : Après guerre : préparation et réorganisation de l'économie française 1914-1921 et F/12/ 8045 à 8062 : Comité consultatif des arts et manufactures. Rapports et mémoires en vue de la publication d'un rapport général sur l'industrie française. L'exploration des archives sera complétée par celle du fonds « Clémentel » conservé aux Archives départementales du Puy-de-Dôme. celle d'une légitimation de l'extension des prérogatives du département du commerce, de l'exigence des administrateurs techniques de l'État de s'affirmer comme les acteurs essentiels de la reconstruction industrielle. Ce rapport marquel'évolution d'une pensée et de pratiques, celle de l'action gouvernementale en matière d'interventionnisme qui se concrétise par la création d'un véritable ministère de l'économie industrielle.

Au-delà de son statut d'archive, l'intérêt du document réside donc dans l'explicitation des conditions de sa production et des motivations qui président à sa réalisation. Au-delà des chiffres et de leur rhétorique s'expriment des enjeux politiques, des justifications de réforme des cadres d'intervention de l'Éłat, une volonté de renégocier les termes de la question sociale, l'affirmation d'une culture de gouvernement qui s'élabore au fil de l'action. Le rapport général sur l'industrie française s'avère être au final l'une de ces manifestations remarquables du désir d'édifier une économie rationnelle qu'appellent de leurs vœux les partisans d'une technocratie auxquels le Ministre Étienne Clémentel s'est allié pour la circonstance. Ce qui fait de ce document technique et administratif un objet de l'histoire non seulement économique, mais tout autant de l'histoire politique de la France en guerre, de l'histoire intellectuelle, sociale et culturelle de la $\|^{e}$ République, et plus précisément de l'un de ses épisodes technocratiques les plus intense.

La reproduction de l'introduction de 1919 proposée dans les pages suivantes est une incitation à lire ou relire ce document suivant au moins trois registres de lecture. D'abord une lecture guidée par son statut de rapport administratif composé de données techniques et de recommandations, dont certaines ont vocation à se traduire sous la forme de mesures législatives immédiates. Ensuite la lecture d'un document dont les conditions de sa production invitent à le considérer comme l'aboutissement d'une entreprise de consolidation des attributions que s'est octroyé le ministère durant la guerre. Enfin une lecture guidée par la dimension sociale et culturelle d'une proposition de refondre une politique industrielle sous l'égide de la rationalisation. Les travaux de ce groupe de techniciens sont alors à considérer comme ceux d'une institution chargée de produire une expertise indispensable à la définition d'une politique de l'État.

Agissant comme rouage ou comme blocage, dans quelle mesure ce lieu de la technocratie politique a-t-il contribué à supporter ou au contraire à mettre en difficulté certaines des perspectives politiques et sociales d'avant guerre ? Le rapport contribue-t-il lui-même aux orientations de l'économie 
et de la réforme sociale dans l'entre-deux guerres? L'équipe d'Étienne Clémentel et la publication de son rapport prolongent en tout cas cette histoire de la "nébuleuse réformatrice " d'avant guerre, contribuant à redéfinir l'horizon des possibles et à rénover les cadres de l'action publique ${ }^{5}$. Le rapport d'Étienne Clémentel mérite assurément une analyse plus approfondie. Elle ne peut être ici qu'esquissée au travers de commentaires sur son introduction générale et la suggestion d'une lecture plus avisée du document dans son intégralité.

\section{Un document pour l'histoire de l'économie industrielle} Une synthèse programmatique

Au premier abord, cet imposant document produit par les services du Ministre du commerce et de l'industrie est la synthèse des réflexions que cette administration a mûries durant le conflit sur la façon dont la nation devait relever les défis qu'elle s'était imposés en s'engageant dans la guerre.

La démarche qui consiste à établir une synthèse en guise de bilan n' a cependant en soi rien d'original. Les études sur la production et le commerce, avant et pendant la guerre, accompagnées de recommandations pour en améliorer les performances sur la scène internationale, sont un exercice auquel s'est astreinte chacune des administrations de guerre. Non seulement des instances publiques, mais aussi des regroupements d'intérêts économiques et industriels se sont attelés à la tâche, motivés en grande partie par la menace de voir le gouvernement contraindre en matière d'économie. Fonctionnaires, experts et porte-parole d'intérêts particuliers se sont dans ce but réunis et ont publié d'innombrables propositions de solutions pour l'avenir'. Parmi les organisations concernées on trouve l'Union nationale pour l'exportation des produits français, la Commission Méline ou encore

5 Christian Topalov, "le champ réformateur, 1880-1914: un modèle " dans Christian Topalov éd., Laboratoires du nouveau siècle. La nébuleuse réformatrice et ses réseaux en France (1880-1914), Paris, éd. Ehess, 1999, pp. 462-474; Janet Horne, Le musée social. Aux origines de l'État providence, Paris, Belin, 2004.

6 Georges Hersent, Une politique de la construction après la guerre : Travaux publics et bâtiment, Paris, Payot, 1919 , 311 p. ; Hélie Mothes, Le commerce et l'industrie après la guerre, Tonneins, éd. de l'imprimerie Ouvrière, 1917, 16 p. ; David Siderski, Contribution au progrès industriel français de I'après-guerre, industrie chimique, matières colorantes, brevets, Paris, H. Dunod, 1916, 48 p. ; Charles Gaujoux, L'effort de l'industrie française pendant la guerre, Thèse de l'Université de Montpellier, Impr. L'Abeille, 1920, 216 p. ; Victor Cambon, Notre avenir, Paris, Payot, 1916, $280 \mathrm{p}$.
I'Association nationale d'expansion économique (ANEÉ). Cette dernière est fondée en décembre 1915 par le président de la Chambre de commerce Arthur David-Mennet. Ébauche d'une confédération générale du patronat, elle regroupe des chambres de commerces et des industriels dont la vocation est de mener enquêtes et travaux en vue de la préparation économique de l'après-guerre, notamment en se constituant en groupe de pression et d'influence auprès de l'administration et du législateur. Elle annonce dès sa création qu'elle entend établir sur le terrain économique l'Union sacrée déjà réalisée entre les partis politiques. Elle s'engage dans la réalisation d'une enquête nationale sur la production française et la concurrence étrangère'. Arthur David-Mennet et ses partenaires rejoignent toutefois vite l'entreprise d'Étienne Clémentel. Certains des contributeurs à l'enquête seront ainsi les rédacteurs du rapport officiel de 1919. Ils exposent aussi ailleurs et indépendamment de l'administration ce que n'avaient pu contenir les limites fixées par le ministre ${ }^{8}$.

Aucune des publications ne présente néanmoins l'ampleur, la cohérence et l'ambition de synthèse proposée par Étienne Clémentel. Soulignons donc d'emblée qu'aucune publication, bénéficiant à la fois de la caution de l'État et arborant cette monumentalité n'a été produite au cours des hostilités. Ce n'est qu'à l'heure des bilans que les études rétrospectives et les recommandations se multiplient`. La plupart est éditée dans la série

7 Voir Bulletin et Bulletin-circulaire de l'Association Nationale d'Expansion Économique et Enquêtes sur la production française et la concurrence étrangère, Paris, 4 volumes, 1917 dont Henri Hitier, Rapport général sur la Section agricole, Libraires-imprimeurs Réunis, 1917, 71 p. ; Henry Girard, Le matériel agricole en France, Paris, Anneé, 1917, 17 p. ; Paul de Rousiers, Rapport présenté par Paul de Rousiers à la section économique de la Commission des études du statut légal des ententes et des cartels, Paris, Anneé, 1916, 23 p. ; Henri Hauser, Les cartels et les syndicats de producteurs en Allemagne, Paris, 1916, $15 \mathrm{p}$.

8 Tel Arthur Fontaine, La guerre et l'industrie française. L'industrie française pendant la guerre, Paris, Puf, 1925, ou Léon Guillet, L'enseignement technique supérieur à l'aprèsguerre, Paris, Payot, 1918, et avec Jean Durand, L'industrie française : l'œuvre d'hier - l'effort de demain, Paris, Masson, 1920 ; Henri Hauser, Les régions économiques, Paris, B. Grasset, 1918 (préface de Étienne Clémentel). On retrouve ainsi dans les dossiers du ministère les éléments ayant servis à la confection de ces ouvrages publiés en marge du rapport Clémentel. 9 Trustee, Le bilan de la guerre, Paris, 1921 ; Maxime Leroy, Pour gouverner, Paris, Grasset, 1918 ; George Guy-Grand, Le conflit des idées dans la France d'aujourd'hui (Trois visages de la France), Paris, M. Rivière, 1918 ; Roger Franca, 


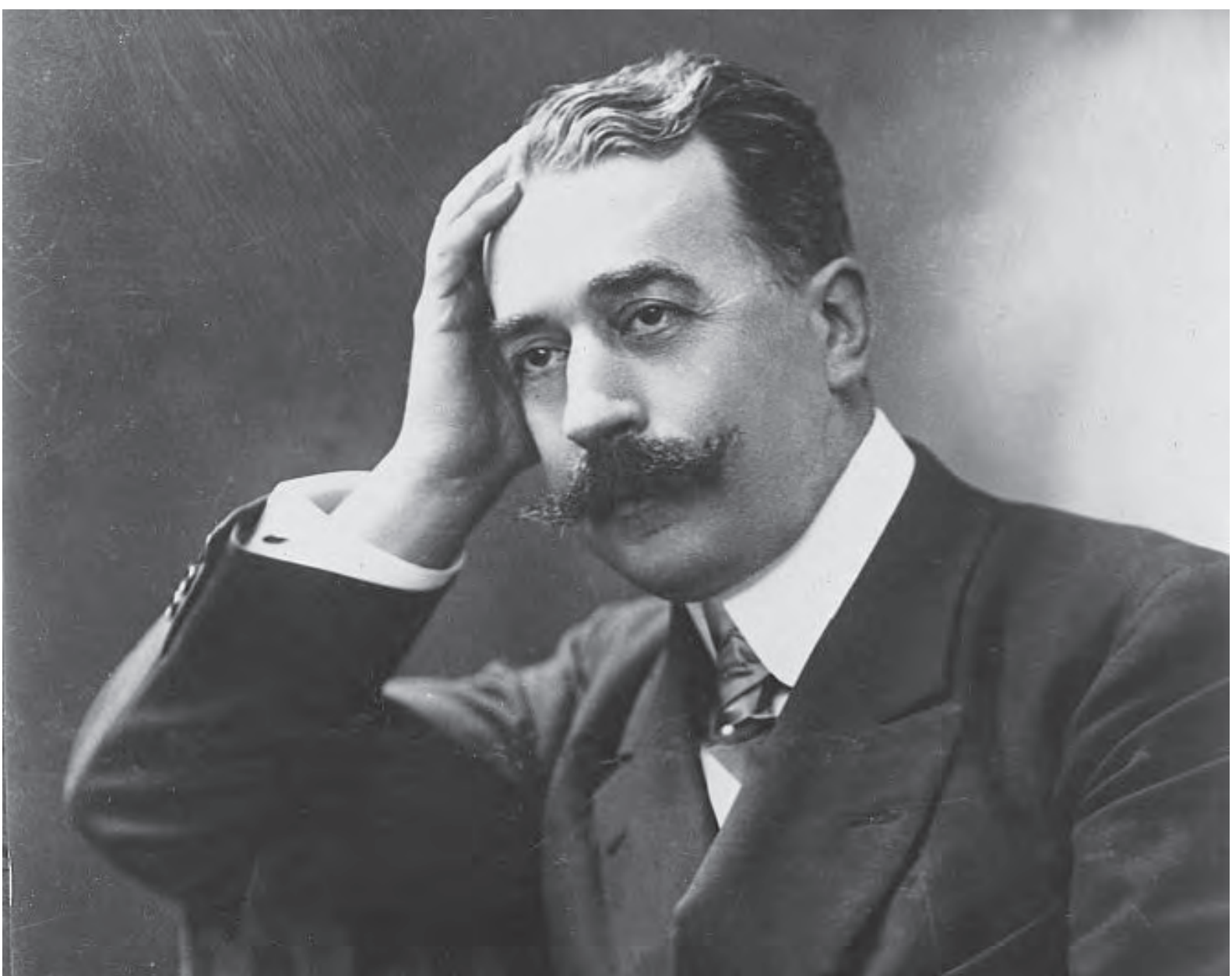

Étienne Clémentel (1864-1936) - George Grantham Bain Collection (Library of Congress) [Public domain], via Wikimedia Commons

française des publications de la fondation Carnegie pour la paix internationale, et en particulier les volumes dédiés à la situation des nations pendant la guerre qui livrent quelques propositions pour un avenir déjà plus ou moins périmé ${ }^{10}$. Elles constituent " un repère pour l'histoire économique de la guerre" "I.

Le travail au pouvoir. Essai d'organisation technique de l'État démocratique, Paris, Éd. de la Sirène, 1920 ... parmi les innombrables réflexions qui fleurissent au sortir de la guerre. 10 James Thomson Shotwell éd., Social and Economic History of the World War, New York, Carnegie Endowment for International Peace, 1921-1937, 150 vols. On y trouve le fameux Pierre Renouvin, Les formes de gouvernement de guerre, Paris, Puf, 1925. Pour une présentation complète de l'entreprise et la liste des 37 références constituant la série française : Alain Chatriot, "Une véritable encyclopédie économique et sociale de la guerre. Les séries de la Dotation Carnegie pour la paix internationale (1910-1940) II, L'Atelier du centre de recherches historiques (revue électronique du CRH sur revue.org), 2009. 11 Antoine Prost et Jay Winter, op. cit., 2004, p. 146. Villes françaises (Lyon, Bordeaux, Tours, Bourges, Rouen, Marseille, Paris), secteurs d'activités ou industries particulières (forces
Le rapport y aurait pourtant eu sa place. II aura été en ce cas trop précoce. Mais c'est justement cette production "à chaud " qui lui confère sa singularité. Elle permet d'examiner la façon dont non seulement le gouvernement pense organiser l'économie au moment où elle est soumise à des contraintes exceptionnelles, mais également les moyens envisagés pour la transformer et que commandent ces mêmes circonstances. II ne fait dès lors pas de doute que ce rapport n'aurait pu exister sous sa forme originale s'il avait été rédigé après-guerre. De fait, le décalage est net entre les énoncés de départ et leur livraison terminale. Comme d'autres agents gouvernementaux de

hydrauliques par exemple) font ainsi l'objet d'études dédiées, mais l'ambition n'est pas celle d'une vaste synthèse. Étienne Clémentel y publie d'ailleurs lui même, La France et la politique économique interalliée, Paris, Puf et New Haven, Yale university press, Publications de la Dotation Carnegie pour la paix internationale, 1931, essai où il expose sa conception de l'organisation du commerce mondial, tout en prenant soin de ne pas adopter la posture de témoin au titre d'administrateur de l'économie française durant la guerre. 
l'économie de guerre, Étienne Clémentel refoulera dès 1919 des tentations interventionnistes qui se sont exprimées sous le coup de l'urgence et de la pénurie. Il occulte des velléités encore proches d'un étatisme, d'une forme d'ingérence qui ne peut se dédire'2. L'introduction au rapport rédigée en 1919 évoque la "dictature de l'État dans l'intérêt suprême de la défense nationale ", mais qui "n'a pas survécu aux circonstances qui l'avaient fait naître ". Clémentel n'hésite d'ailleurs pas à revenir en 1931 sur cette menace de " dictature économique de l'État" dont il a été l'un des protagonistes, mais estime qu'elle a été le pire des risques auquel s'est exposé le pays pendant la guerre ${ }^{13}$.

L'emprise administrative de l'État sur l'économie n'est cependant pas ce qui le préoccupe le plus. Son Rapport général sur l'industrie française, sa situation, son avenir est converti dès les premières lignes en "rapport général sur l'Organisation de la Production nationale au lendemain de la guerre " (notez les termes affublés d'une majuscule). Étienne Clémentel précise ainsi et annonce d'emblée ce dont il est véritablement question. Après un constat des plus alarmistes sur la situation française devant susciter l'angoisse et l'urgence d'agir, l'introduction tempère vite les inquiétudes pour laisser place à l'action : la mise en œuvre d'un programme de réorganisation rationnelle de la production industrielle. Léon Guillet, directeur des services techniques, et pour l'occasion responsable de la coordination du rapport, rappelle dans sa propre présentation que ce rapport "est en somme la synthèse des travaux poursuivis en vue de la réorganisation industrielle du pays ${ }^{14} \|$. Le salut de l'économie française passe donc parl'organisation, dont la mention apparaît une vingtaine de fois dans le texte, associée aux termes "doctrine ", "principe " et "programme " (14) ${ }^{15}$. La France a besoin d'une ligne de conduite, d'un guide pour l'action, et plus encore comme l'indique plus loin Étienne Clémentel, d'une doctrine économique.

12 Fabienne Bock, op. cit. 1984.

13 Étienne Clémentel, op. cit. 1931.

14 Léon Guillet, 1919, vol. 1, xxxii.

15 Les principales occurrences sont assez logiquement « industrie " (71), " production " (60) et " commerce " (59). Les termes ou les expressions renvoyant au domaine administratif (réforme, gouvernement, administration, ministère, contrôle, enquête et études) totalisent 40 mentions. " capital " et " capitaux " rassemblent 21 occurrences, " travail » 11, mais « démocratie » et « social » ne sont mobilisés respectivement que 1 et 2 fois sur la totalité du texte.

\section{Rationaliser l'organisation}

Le rapport est défini dans son introduction comme celui d'un comité d'experts indiquant les moyens à mettre en œuvre pour relever la France. Ce programme de gouvernement soumis au Parlement se présente sous la forme d'une série d'avis qui se veulent solidement établis sur la base d'études scientifiques et techniques. Ils s'énoncent dès lors comme des solutions nécessairement valides et légitimes, et que les politiques se doivent de considérer le plus sérieusement du monde.

Les termes sont clairs. II faut « accroître dans toute la mesure du possible notre productivité ॥. Pour ce faire, Clémentel propose « un corps complet de doctrine économique (...) ce corps de doctrine forme en réalité un grand programme de l'œuvre à accomplir 1. . C'est à une mise au travail de l'ensemble de la population à laquelle Étienne Clémentel invite, petits rentiers et travailleurs. II réserve toutefois aux femmes la direction du foyer et la fonction de remplir les berceaux, signifiant au passage le danger que comporte la mobilisation de la main-d'œuvre étrangère : la dégénérescence de la race qu'il faut protéger. On trouvera ainsi dans la dernière partie du rapport (les méthodes d'expansion économiques) une série de propositions des plus conservatrices pour les femmes, et ailleurs pour les indigènes des colonies, les travailleurs, les enfants des classes populaires ou les subalternes. À l'instar de la plupart des propositions associées aux thèmes sociaux, les avis experts usent du langage de la rationalité instrumentale, occultant leur caractère politique pour s'affirmer comme incontestables.

Le machinisme est ensuite présenté comme I'horizon indépassable de l'économie rationnelle, la panacée pour surmonter toutes les difficultés présentes et à venir : "dans l'usine modernisée, les agents d'exécution seront capables d'obtenir un plein rendement du machinisme le plus perfectionné, créé par nos élites scientifiques et installé par des chefs d'entreprise audacieux et novateurs ", mais "qu'une distribution rationnelle des fonctions individuelles dans l'œuvre collective ॥) viendra assurer l'efficacité. C'est là le domaine de la division sociale du travail et de l'organisation scientifique de la production industrielle où «l'effort de réflexion est fait à l'origine par les ingénieurs», et où "le manœuvre d'antan vient remplir son rôle en collaborateur souple et intelligent $"$. Dans cet univers tout mécanisé et si harmonieux, la "standardisation » et le "travail en série " sont des impératifs de gouvernement rationnel que permettent les "laboratoires " et les "études expérimentales ॥, l'alliance de la science et de l'industrie : "le savant doit sortir de sa tour d'ivoire 
et l'industriel s'élever au dessus des préoccupations immédiatement utilitaires (...) Les nécessités de la guerre ont mis en contact le savant et l'industriel ; désormais ils ne s'ignorent plus ». Non seulement les énergies humaines sont mobilisés dans ce grand dessein, mais aussi les énergies fossiles et celles produites par les centrales électriques. Toute une série de solutions techniques mais aussi législatives sont préconisées afin d'optimiser leurs usages. L'agriculture est également soumise à l'injonction de la mécanisation et à l'utilisation des engrais chimiques que la France peut désormais produire en abondance.

Par la suite, Étienne Clémentel pointe quelques unes de ce qu'il juge comme des défaillances de la société française et de ses membres : «le capitalisme français timide et conservateur ", la réticence des industriels à constituer des groupements d'intérêts et une difficulté à se convaincre que "l'initiative collective est une forme supérieure de l'initiative privé ") ; la grande volatilité de l'épargne des nationaux plus enclins à investir au-delà des frontières; le manque de discipline ou encore une réticence certaine à l'asservissement volontaire. Reprenant les propos de Waldeck-Rousseau "il faut que le capital travaille et que le travail possède ", Étienne Clémentel en appelle à la "collaboration intime du capital et du groupement professionnel, grâce à laquelle l'ouvrier sera intéressé à la production ॥. Ces résultats, Étienne Clémentel les envisagent dans le cadre d'une "liberté organisée », euphémisme pour dire qu'il souhaite fermement " assurer cette collaboration des pouvoirs publics et des forces de production " et "indiquer aux industriels, aux commerçants, ou plutôt aux groupements qui les représentent, le plan général suivant lequel ils doivent diriger leurs efforts pour que l'action des particuliers vienne aider et non entraver celle de l'État ॥. Cette collaboration, Étienne Clémentel la conçoit sous l'égide d'une confédération des Chambres de commerce permettant « de grouper les forces éparses sur le territoire, de les associer dans une action commune et de leur donner, dans les centres qui ont un pouvoir de rayonnement régional, une représentation qui leur permet de devenir d'utiles auxiliaires du pouvoir central dans l'œuvre qu'il poursuit "l

Voilà en quelques mots d'une synthèse de la synthèse le ton et la teneur des objectifs que se sont fixés les tenants de l'organisation rationnelle de la production en vue du relèvement de l'économie nationale.

\section{Naissance d'un ministère de l'économie rationnelle} Urgence et dépendance

Aucune mobilisation industrielle et économique n'avait été pensée, moins encore planifiée pour anticiper un conflit de cette nature ${ }^{16}$. Au déclenchement des hostilités, le sénateur Charles Humbert peut ainsi clamer au Palais le 13 juillet 1914, lors de l'examen de projets concernant les dépenses relatives à la défense nationale, qu' "il manque deux millions de paires de chaussures ! ${ }^{17} \|$. La panique manifeste traduit surtout le constat d'une impréparation économique et financière peu raisonnable, attisée par la conviction que l'Allemagne s'est dotée d'un « trésor de guerre » constitué durant plusieurs années ${ }^{18}$.

Dès l'automne 1914, la guerre enlisée devient une épreuve de force économique autant que militaire. La conviction s'était installée parmi les puissances en conflit que la victoire devait dépendre de leur capacité à mobiliser la nation industrielle dans l'effort de guerre ${ }^{19}$. Amputée d'une bonne partie de ses ressources minières et de ses usines métallurgiques, la France se trouve sur ce registre en bien mauvaise posture ${ }^{20}$. Le terme de désastre n'est pas exagéré pour qualifier les pertes en capacités matérielles, en ressources naturelles, industrielles et humaines. Elles imposent la gestion des urgences les plus immédiates. À cela s'ajoutent des difficultés d'approvisionnement en fournitures provenant d'Allemagne dans de nombreux domaines stratégiques (machine outil, verrerie, brique réfractaire, etc. $)^{21}$.

L'industrie française s'organise de façon empirique. La prolongation des hostilités et la désorganisation de la production imposaient de toute façon la refonte des plans d'approvisionnement en

16 Gerd Hardach, "La mobilisation industrielle en 19141918 : production, planification et idéologie », dans Patrick Fridenson (éd.), 1914-1918, l'Autre front, Cahier du " Mouvement social $11, n^{\circ} 2$, éd. ouvrières, 1977, pp. 81-109; JeanJacques Becker, 1914. Comment les français sont entrés dans la guerre, contribution à l'étude de l'opinion publique, printemps-été 1914, Paris, FNSP, 1977 ; Marc Ferro, La Grande guerre 1914-1918, Paris, Gallimard.

17 Georges Bonnefous, Histoire politique de la Troisième République.t. 2. La grande guerre (1914-1918), Paris, Puf, 1967, p. 17. 18 Georges Bonnefous, op. cit. 1967, p. 70.

19 Richard F. Kuisel, op. cit., 1984, p. 73.

20 Voir dans le rapport lui-même les déficits de production et d'importation de fer, charbon et acier entre 1913 et 1918, que l'on trouve aussi dans Arthur Fontaine, op.cit., 1925.

21 Archives nationales F/12/8039 et 8041 ; Marjorie M. Farrar, Conflict and Compromise: The Strategy, politics, and Diplomacy of the French Blockade, 1914-1918, La Hague, Martinus Nijhoff, 1974. 
armes et des réquisitions. Mais le conflit rendait aussi difficile le fonctionnement normal de l'économie de marché. La puissance publique est dès lors conduite à adopter une gestion plus autoritaire des relations entre le gouvernement et les agents économiques du pays. Le ministère du commerce exerçait traditionnellement à l'ombre des Affaires étrangères et se contentait de gérer les intérêts nationaux au travers des importations et des exportations. II va désormais s'instaurer comme l'un des éléments clé d'une organisation administrative où s'inaugure l'intervention économique directe. II lui est assigné, pour la première fois, la fonction d'un ministère de l'économie nationale.

\section{Étienne Clémentel aux commandes}

Après la cascade des déclarations d'hostilité en août 1914, la vie politique française s'apprête à concilier le maintien du régime républicain et un mode de gouvernement en temps de guerre. Dès le 26 août, le président du Conseil René Viviani élargit l'union sacrée en remaniant son gouvernement ${ }^{22}$. La vie parlementaire ne reprend cependant qu'après la bataille de la Marne, en fin d'année, avec deux préoccupations majeures : régler les rapports entre les pouvoirs civils et militaires; trouver les moyens de financer une guerre qui s'est à l'évidence installée pour durer ${ }^{23}$. Alexandre Millerand, ministre de la guerre, se voit par exemple imposer le 18 mai 1915 quatre secrétaires d'État, dont le socialiste Albert Thomas, chargé de l'artillerie et de l'équipement militaire. Quelques mois plus tard, Viviani cède la présidence à Aristide Briand. C'est ce dernier qui appelle le 29 octobre 1915 le député radical Étienne Clémentel au ministère du commerce et de l'industrie.

Né le 29 mars 1864, Étienne Clémentel a entrepris à la faculté de droit de Clermont d'être notaire à 25 ans. Assez classiquement, il bifurque vers la politique. De la position de secrétaire auprès du sénateur du Puy de Dôme et ministre de l'agriculture, Étienne Clémentel s'installe en 1901 dans la position de député de Riom ${ }^{24}$. Sa carrière ministérielle, elle, débute en 1905 avec le portefeuille des colonies. Le ministère de l'agriculture suit en 1913. C'est là que le président du Conseil Aristide Briand vient le trouver.

22 Sur la vie politique de la République en guerre : George et Édouard Bonnefous, Histoire politique de la Troisième République : 1914-1918. La grande guerre, volume 2, Paris, Puf, 1967. 23 Jean Jacques Becker et Serge Berstein, op. cit., 1990, "Gouverner en guerre », pp. 52-85.

24 Guy Rousseau, Étienne Clémentel, 1864-1936 : entre idéalisme et réalisme, une vie politique : essai biographique, Archives départementales du Puy-de-Dôme, 1998.
Qu'est-ce qui peut justifier de confier à ce parlementaire plutôt conventionnel, sans expérience notoire de gestion des affaires industrielles, l'un des principaux portefeuilles ministériels d'un gouvernement en guerre ? II n'est pas considéré par ses pairs comme un homme particulièrement exceptionnel, mais plutôt qualifié de fonctionnaire compétent et rigoureux. De surcroît, Étienne Clémentel est un poète, un artiste sculpteur, un peintre qui n'a jamais cessé de peindre, un auteur de pièces de théâtre, intime des milieux artistiques, bref il est ce qui semble ne pas devoir le désigner spontanément pour occuper les plus hautes fonctions techniques en période de guerre et de crise. A son arrivée, Étienne Clémentel n'a en effet pas de programme politique clairement affiché, ni plan économique, ni doctrine. Pour tout dire, il n'est pas vraiment le partisan emblématique d'une politique qui sera la sienne. Son action gouvernementale le conduit néanmoins à mettre en œuvre un véritable programme de gestion inédite d'un état entrepreneur industriel. II semble que ce soient, sinon la dimension politique et la recherche d'un équilibre entre les partis qui ont guidé le choix de Briand $^{25}$, peut être au moins sa fonction au début de la guerre de missionnaire au nom du gouvernement auprès des producteurs d'obus et d'autres industriels susceptibles de convertir leurs équipements ${ }^{26}$.

Au côté du ministre de l'armement, Étienne Clémentel constitue l'un des centres névralgiques de la bureaucratie gouvernementale ${ }^{27}$. E† c'est dans cette fonction qu'il semble avoir marqué ses contemporains. Ses collaborateurs et amis reconnaîtront, dans un hommage collectif et anonyme publié en 1932, que " c'est à coup sûr dans cette position de ministre du commerce que Clémentel donne la plénitude de sa valeur, de sa capacité de travail et de sa large intelligence ${ }^{28} \%$. Son rapport de 1919 est censé en témoigner. II semble en effet qu'il le considère comme l'une de ses réalisations importantes, ce que démontrerait l'investissement personnel consenti. II fait tout ce qui est en son pouvoir pour maintenir la continuité de l'exercice pour lequel il affirme devoir disposer de temps. II négocie avec Édouard Herriot de mener à terme la mission dont il s'est investi. II conserve ses attributions en 1916 ; rejette en 1917 la proposition d'Alexandre Ribot de reprendre le portefeuille des

25 Clothilde Druelle, op. cit., 1993, p. 7.

26 Sur l'arrivée de Clémentel : George-Henri Soutou, op. cit. pp. 234-236 ; Clothilde Ruelle, op. cit. 2004, pp. 31-65.

27 Guy Rousseau, op. cit. 1993, pp. 139-160.

28 Collectif, Étienne Clémentel, Paris, Librairie de France, 1932. Clémentel sera de nouveau Ministre en 1924, mais des finances. 
finances. En dépit des difficultés auxquelles le projet se trouve maintes fois confronté, jusqu'à parfois remettre en cause sa conclusion, Étienne Clémentel souhaite fermement garantir sa publication et sa diffusion. II le considère assurément comme un travail de la plus haute importance pour l'avenir du pays.

Étienne Clémentel est donc de ceux à qui le gouvernement confie la mission d'organiser l'économie de guerre. Aux côtés d'Albert Thomas et de Louis Loucheur, tous deux ministres de l'armement, Étienne Clémentel domine à la fois la politique économique et contribue à délimiter ses contours dans l'après-guerre. Après l'armistice, son collègue Louis Loucheur est ministre de la Reconstruction industrielle. Étienne Clémentel dirige, lui, son ministère sans rupture jusque fin 1919. Pourquoi cependant le ministère du commerce s'était-il investi d'une telle mission, alors même que cette administration n'avait eu jusqu'alors qu'un rôle bien modeste dans l'organisation économique du pays avant guerre? Quels sont les motifs, les moyens et les cadres d'action dont se dote ce ministère pour finalement devenir l'un des agents essentiels de la transformation des structures économiques, sociales et politiques de l'après-guerre ? La lecture et l'histoire de ce rapport livre indéniablement à ce propos quelques éclaircissements.

Éternel complexe français et guerre économique Après la défaite de 1870, la France avait prise l'habitude de mesurer le déclin de son industrie à l'aune de la production, du dynamisme industriel, du commerce et de la qualité des produits allemands ${ }^{29}$. Cette supériorité économique et commerciale que démontre l'occupation du territoire français par les armées du Reich, les contemporains d'Étienne Clémentel l'ont largement attribué tant aux capacités exceptionnelles d'organisation de la nation allemande que de l'indiscipline des français trop épris de liberté ${ }^{30}$. Tel cet auteur qui dans deux

29 Voir par exemple l'alerte bien connue concernant le retard français dans le domaine de l'industrie chimique lancée par Albin Haller à l'occasion de l'Exposition universelle de Chicago : « La lutte des nations sur le terrain de l'industrie chimique ॥, Revue Générale des Sciences, 5, 1884, pp. 473-487 ; voir aussi Maurice Schwob, Le danger allemand : étude sur le développement industriel et commercial de l'Allemagne, Paris, Chailley, 1896 et Avant la bataille, Paris, Flammarion, 1904.

30 Claude Digeon, La crise allemande de la pensée française, Paris, Puf, 1959 ; Albert Sauveur, L'Allemagne et la guerre Européenne, Paris, Bloud et Gay, 1916 ; Victor Cambon, L'Allemagne au travail, Paris, P. Roger et Cie., 1909 ; Henry Le textes rédigés avant et après guerre reproche aux élites françaises de n'avoir jamais su, à la différence de l'Allemagne, se donner les moyens de concilier les divergences nationales derrière l'intérêt commun d'une industrie encadrée par l'administration ${ }^{31}$. Dans sa préface de 1915 à la traduction de l'ouvrage du général Friedrich von Bernhardi, Georges Clémenceau pouvait ainsi résumer la conviction partagée par les élites françaises d'un monde économique soumis au péril germanique ${ }^{32}$ : «Il y avait un danger allemand, un danger plus redoutable dans la paix que dans la guerre... Par la haute valeur d'un prodigieux effort de travail méthodique, dans une savante organisation de machinerie humaine, ces gens étaient en train de conquérir le monde". C'est un point sur lequel avait comme bien d'autres insisté Henri Hauser, universitaire à la Sorbonne et proche de Clémentel, louant l'esprit de discipline du peuple allemand et de ses industriels, l'efficacité de leur système de production et leur aptitude à l'organisation rationnelle, et dont bien évidemment les français se devaient de s'inspirer au plus vite ${ }^{33}$. Étienne Clémentel a par ailleurs connaissance d'une étude commandée en 1913 par le ministre du commerce allemand sur l'implication de son industrie en cas de conflit ${ }^{34}$. Comme beaucoup d'autres, sa

Chatelier, « Du rôle de la science dans la lutte contre l'industrie allemande "), Bulletin de la Société d'Encouragement pour I'Industrie Nationale, 22, 1915, pp. 174-180.

31 « il leur eût fallu pour cela de l'énergie, de la méthode, et un esprit national ; il leur eût fallu le sentiment, qui ne manquait pas en Allemagne, des devoirs de l'industrie et de l'argent envers le pays. Cet esprit, on ne le trouvait pas. Une insuffisante application du travail scientifique aux industries, une timidité peureuse qui hésitait à courir les risques ... $\|$ : George Guy-Grand, Le procès de la démocratie, 1911, p. 110, et dix ans plus tard : " Là-bas, outre-Rhin, une de ces fortes passions menait le peuple, un impérialisme puissant, aux vues gigantesques, qui enflammait les lourdes imaginations d'un délire de grandeur. Et la présence constante de cette volonté de conquête stimulait les administrations, soutenait les savants, fouettait les industries, établissait entre toutes les techniques une collaboration et une coordination continuelles, et multipliait ainsi prodigieusement les forces de l'empire " Le conflit des idées dans la France d'aujourd'hui. (Trois visages de la France), Paris, Marcel Rivière, 1921, p. 108. 32 Préface de Georges Clémenceau de la traduction de Friedrich von Bernhardi, Notre avenir: les ambitions allemandes et la guerre, Paris, L. Conard, 1915.

33 Henri Hauser, Les méthodes allemandes d'expansion économique, Paris, A. Colin, 1915 et « L'Allemagne économique. L'industrie allemande considérée comme facteur de guerre "I, Bulletin de la Société d'Encouragement pour I'Industrie Nationale, 114, 1915, pp. 425-439.

34 Pierre Renouvin, La crise Européenne et la Grande guerre, 
conviction est que l'Allemagne engagerait sur le terrain économique une offensive majeure après les hostilités ${ }^{35}$. La métaphore guerrière est dès lors de circonstance dans le rapport de 1919. Le Ministre prône la mobilisation des techniques militaires pour la conquête des marchés commerciaux ; la bataille doit se poursuivre sur le terrain de l'économie, car la lutte économique, rappellet-il, n'est que la poursuite de la guerre par d'autres moyens. "Voici donc nos capitaines d'industrie entourés d'une incomparable armée au travail ") : il fallait s'organiser et se discipliner pour gagner la dernière manche, se dispenser de commercer avec les nations adverses, mais plus encore s'emparer des marchés ennemis. Telles sont les annonces d'Étienne Clémentel faites à son arrivée.

L'Allemagne a pourtant bien été confrontée à de rudes difficultés pour s'organiser économiquement durant le conflit. Certes la gestion de l'économie de guerre allemande fournit un bon exemple de I'un des complexes militaro-industriels précoce, mais qui s'avère surtout peu efficace. Face à la guerre totale, sa politique est en réalité défaillante ${ }^{36}$. Mais ce n'est pas là l'essentiel pour les défenseurs français d'un nouvel ordre rationnel de l'économie. La démonstration du bel exemple d'une Allemagne parfaitement organisée, de sa capacité supposée à la discipline industrielle, de son aptitude en matière de dirigisme et d'expansion de son commerce d'avant-guerre devait bien servir la cause des partisans français de l'efficace sous l'égide de la rationalisation.

\section{Les cadres de l'action}

Étienne Clémentel s'est donc rapidement convaincu de devoir investir plus franchement le champ économique et de la production. Dès sa prise de fonction, il propose un plan de mobilisation de l'économie civile au service de la nation. II diffuse des circulaires dans ce sens, produit des discours invitant les industriels à mettre en œuvre, sans plus tarder,

1904-1914, Paris, Puf, 1969, p. 283 ; Raymond Poidevin, Les relations économiques et financières entre la France et I'Allemagne de 1898 à 1914, Paris, A. Colin, 1969.

35 Robert Ducasble, Le Développement mondial du commerce allemand de 1894 à 1913. Comment la France doit se préparer à la lutte commerciale de l'après guerre, Paris, Imp. Technique, 1917, 19 p. ; Siegfried Herzog, Le plan de guerre commerciale de l'Allemagne, Paris, Payot, 1919 ; Norbert Lallié, La guerre au commerce allemand, Paris, Recueil Sirey, 1918. 36 Gerald D. Feldman, Army, Industry and Labour in Germany 1914-1918, Berg Publishers, 1992, pp. 253-300. les principes de l'organisation scientifique du travail. Dans son « Projet de réorganisation des services du ministère du commerce et de l'industrie», il se dit en 1916 conscient de la nécessité de l'adhésion de l'industrie française à un plan d'action indiqué par les autorités en accord avec les intérêts de la nation et de sa défense ${ }^{37}$. Le ministère du commerce fit dès lors un premier pas vers le rééquipement de l'appareil productif national et une meilleure autosuffisance en entreprenant un inventaire général des capacités industrielles de la nation. Il demande à ses services techniques et au Comité Consultatif des arts et manufactures de déterminer, en liaison avec les industriels, les besoins de chaque secteur, de formuler des recommandations pour l'amélioration de la productivité.

Avantqu'Étienne Clémentel ne prenne en mains le ministère, l'intervention de l'État s'était toutefois déjà manifestée par le contrôle de la production, notamment au travers des commandes et du paiement, des avances sur la construction des usines, de la répartition des matières premières, des moyens de transport maritimes placés sous sa dépendance. Une loi du 16 octobre 1915 autorise même le gouvernement à réquisitionner le blé, la farine et le charbon pour la population civile. Étienne Clémentel dispose donc d'emblée d'un potentiel d'intervention, facilitée par l'effacement de l'autorité parlementaire sur le gouvernement. La détention de plusieurs portefeuilles ministériels lui confère par ailleurs une position indiscutablement confortable. En plus du commerce et de l'industrie, il dispose, selon les remaniements successifs, des postes et télégraphes, de la marine marchande, de l'agriculture, de l'aviation, de l'éducation technique et du travail.

Au ministère du commerce et de l'industrie donc de concevoir les orientations de la production et de l'économie. Aux acteurs industriels d'exécuter. C'est le principe de base du plan. Les services techniques s'organisent autour de sections spécialement chargées d'étudier les questions les plus urgentes dans les domaines de la production et des importations pour les produits les plus utiles à l'économie de guerre. Au nombre de dix en 1916 , ces sections s'étoffent pour doubler au fil des mois et traiter de nouvelles questions, et notamment celle des études sur l'après-guerre, en liaison avec le Comité Consultatif des arts et manufactures qui

37 «Services techniques organisés par la loi du 30 mars 1916 », Archives nationales, F/12/7657. 
est chargé de produire la trame principale du plan de réorganisation industrielle proposé en $1919^{38}$.

Réactiver le Comité consultatif des arts et manufactures Le Comité Consultatif des arts et manufactures avait été officiellement institué en 1861, mais c'est en 1880 qu'il est réellement mobilisé auprès du ministère de l'agriculture et du commerce. II est alors « chargé de l'étude et de l'examen de toutes les questions intéressant le commerce et l'industrie qui lui sont renvoyées par le ministère, en vertu des lois et règlements, ou sur lesquelles le ministre juge utile de le consulter, notamment en ce qui concerne : les établissements insalubres; les brevets d'inventions; l'application ou la modification, au point de vue technique, des tarifs et des lois de douane. II peut être chargé de procéder aux enquêtes ou informations qui sont jugées nécessaires par le ministère pour l'étude des questions ci-dessus ${ }^{39} .11$

Ce comité est composé de seize membres pris dans le Conseil d'État, l'Académie des sciences, les corps des ponts et chaussées et des mines, le commerce et l'industrie. La nomination se fait par décret. Le président est désigné chaque année par le ministre. Le comité se réunit au moins une fois par semaine.

Le 4 avril 1917, Étienne Clémentel accorde à cette instance des fonctions et des prérogatives qu'elle n'avait jamais eves. II en remanie la composition pour lui adjoindre tout ce que compte d'autorité scientifique et technique disponible à ce moment : Albin Haller, Gabriel Chandèze, Henry Le Chatelier, Arsène d'Arsonval, Léon Guillet, Lovis de Launay, Auguste Rateau, Louis Lindet, Charles Lauth, Eugène Tisserand, Théophile Schlœsing, Émile Fleurent, André Hillairet, George Charpy, Henri Hitier, Auguste Béhal... . Le ton est donné. Il s'agit bien de constituer un organe d'experts scientifiques et techniques. Sa mission? Centraliser et diriger les études générales concernant l'organisation industrielle du pays pour l'après-guerre et proposer des mesures administratives et législatives en vue de sa réalisation.

38 « tableau récapitulatif des bureaux et sections du ministère du commerce au 24 octobre 1918»Archivesnationales, F/12/7657. Parmi les services techniques fixés par l'arrêté du $j^{\text {er }}$ avril 1916 figurent les sections suivantes : $1^{\circ}$ Métallurgie et forces motrices ; $2^{\circ}$ textiles ; $3^{\circ}$ industrie chimique, verrerie, céramique ; $4^{\circ}$ cuirs et peaux ; $5^{\circ}$ matériel automobile et agricole et industries diverses ; $6^{\circ}$ pétroles, huiles lourdes, combustibles liquides ; $7^{\circ}$ matières grasses ; $8^{\circ}$ papiers ; $9^{\circ}$ récupération et collecte des matières inutilisées ; $10^{\circ}$ Transports commerciaux. 39 Décret du 18 octobre 1880, Archives Nationales, F/12/7657.
Suivant le décret paru pour la circonstance, la direction des études techniques du ministère a désormais dans ses attributions:

La centralisation des travaux du Comité Consultatif des Arts et Manufactures : rapport général - proposition et présentation des mesures d'application,

La mise au point et préparation des mesures d'application des travaux du congrès du Génie civil,

La direction et la centralisation des travaux des commissions de standardisation et d'unification des cahiers des charges. La préparation et la présentation des mesures d'application,

La création des laboratoires nationaux - la préparation et la présentation des décisions relatives,

La participation au point de vue technique aux études relatives à la réorganisation et au développement de l'enseignement technique et professionnel,

La représentation de concert avec toutes les autres directions du ministère du commerce dans la commission d'utilisation des usines de guerre,

La représentation du ministère du commerce à la commission de liquidation des stocks,

La représentation du ministère du commerce à l'office de reconstitution industrielle des régions envahies,

L'étude des questions techniques renvoyées par le ministre et représentation du ministre dans les commissions correspondantes.

Toutes les sections sont tenues, précise Étienne Clémentel, de participer de la façon la plus active aux travaux du Comité Consultatif des Arts et Manufactures. La mission doit se traduire par la publication d'un rapport général sur la situation de l'industrie et du commerce, mais surtout sur les mesures à prendre à l'après-guerre pour en assurer l'expansion. L'équipe s'organise.

\section{Constituer un état-major}

Chaque responsable du gouvernement en charge des affaires économiques s'entoure des compétences dont il estime avoir besoin pour mener ses actions. Compétences qui se sont raréfiées du fait de la mobilisation et de quelques disparitions perceptibles ${ }^{40}$. Chacun recrute là où il peut : parmi les proches et le cercle traditionnel des réseaux de sociabilité, mais dans la plupart des cas, tout simplement parmi les fonctionnaires déjà en poste ou placés dans le giron du ministère, non encore mobilisés bien sûr.

40 Ainsi trois parlementaires avaient été tués lors des combats quand la Chambre des députés se réunit en décembre 1914 : George et Édouard Bonnefous, op. cit. 1967, p. 60. 
Louis Loucheur recrute ainsi chez les polytechniciens ${ }^{41}$. Albert Thomas chez les Normaliens ${ }^{42}$. Étienne Clémentel, lui, chez les Centraliens. Non parce qu'il est particulièrement enclin à mobiliser cette catégorie d'ingénieur, mais parce que l'École centrale est placée sous l'égide du ministère du commerce et que les centraliens sont déjà en place, notamment au sein de la direction technique du ministère. Pour ces mêmes raisons, Étienne Clémentel mobilise autant que possible les fonctionnaires des écoles nationales d'arts et métiers, des écoles pratiques et professionnelles ${ }^{43}$. Mais Étienne Clémentel mobilise d'abord le premier des centraliens, celui qu'il a croisé lors de ses missions conduites auprès des fabricants d'obus au début de la guerre : Léon Guillet, directeur de l'École centrale au moment des faits, et qui se charge de la cooptation de nouveaux collaborateurs. Parmi les centraliens et contributeurs directs au rapport, on retrouve Léon Appert, Paul Biju-Duval, Arnaud Blanc, Adrien Bochet, Emile Boyoud, Joseph Carlioz, Albin Dumas, Georges Dumont, Maurice Ganne, René Guérin, Georges Hersent, André Hillairet, Henri Japy, Pierre Richemond, et bien d'autres encore qui ne sont parfois mobilisés que ponctuellement au titre d'enquêteurs ou de membres des services techniques du ministère.

L'état-major d'Étienne Clémentel est donc recruté en dehors des filières administratives et contraste de fait par sa technicité avec la formation juridique habituelle des fonctionnaires de carrières de ce ministère. Tous ne sont cependant pas centraliens. Étienne Clémentel complète son équipe en mobilisant des cadres de l'administration, des bureaucrates, des experts, des professionnels ${ }^{44}$.

41 Stephen Douglas Carls, Louis Loucheur and the shaping of modern France, 1916-1931, Baton Rouge, Louisiana State university press, 1993, 330 p. et Louis Loucheur, Souvenirs de la Grande Guerre, Lille, Société d'édition du Nord, 1938.

42 Christophe Prochasson, « Entre science et action sociale : le « réseau Albert Thomas » et le socialisme normalien », dans Christian Topalov, Laboratoires du nouveau siècle : la nébuleuse réformatrice et ses réseaux en France, 1880-1914, Paris, EHESS, 1999, pp. 141-158; Martin Fine, « Albert Thomas : a reformer's vision of modernization, 1914-1932 ॥, Journal of Contemporary History, 12 (3), 1977, pp. 545-564 ; Madeleine Rébérioux et Patrick Fridenson, "Albert Thomas, pivot du réformisme français ॥, Le mouvement social, 87, 1974, pp. 85-97; Alain Hennebicque, "Albert Thomas et le régime des usines de guerre (1915-1917) ", dans Patrick Fridenson (dir.), 19141918 : l'autre front, Paris, éd. Ouvrières, 1977, pp. 111-144.

43 « Lettre de Clémentel au Ministre de la guerre, le 6 août 1917 II, Archives Nationales, F/12/7657.

44 Fernand Chapsal avait la haute mission de diriger le ravi-
Pression des alliés, le tournant de 1917

Le sentiment d'une guerre longue, voire interminable, s'était installée. Tout au long de l'année 1917, la communauté nationale traversa une crise à la fois militaire, politique, sociale et morale. Les oppositions croissantes sur les buts de guerre s'exacerbaient. La confiance de la population accordée aux classes dirigeantes s'érodait. L'aspiration à la paix grandissait ${ }^{45}$. Surtout les mouvements sociaux avaient fait leur apparition, bravant l'interdit moral de faire grève en temps de guerre. C'est en novembre 1917 qu'éclate par exemple à Saint-Étienne, première région d'industrie de guerre, un mouvement social qui répond à l'agitation perceptible dans le reste du pays. À la fin de l'année, les échos de la Révolution bolchevique alimente chez certains dirigeants craintes ou fantasmes, sinon d'une contagion révolutionnaire, du moins d'une flambée sociale sans précédent. Par ailleurs, et par crainte de combats imminents et décisifs, les députés acceptent de déléguer aux militaires et au gouvernement ses dernières prérogatives dans la conduite des affaires économiques. Désormais, le gouvernement peut diriger le pays par décrets.

Un autre souci était de lutter contre les spéculateurs qui importaient et stockaient tout ce qu'ils pouvaient dans l'espoir de faire, le moment venu, des profits substantiels, et immobilisaient pour

taillement du pays. Après la guerre il sera sénateur, puis luimême ministre du commerce. Roger Fighiera qui connait le mieux la production reste fidèle à ses hautes fonctions de Directeur des affaires industrielles; Daniel Serruys, un universitaire, avait été appelé à la direction des accords commerciaux. II abandonne plus tard l'administration pour les grandes affaires internationales; Paul Elbel sera le successeur de Clémentel au ministère du commerce : ou encore Louis Leroy, qui fut le chef de cabinet de Clémentel à l'agriculture. D'autres sont des incontournables fonctionnaires en poste, tels Jean Rous, directeur de la répression des fraudes; Henri Tenot, directeur de l'enseignement technique rattaché au ministère du commerce ; Charles Picquenard, directeur au ministère du travail ; Gaston Gourdeau, directeur des services techniques qui est élu député et nommé sous-secrétaire d'état au ministère des travaux publics ; Armand Megglé, directeur du comité national des conseillers du commerce extérieur, considéré comme le disciple de Clémentel par le collectif de ceux qui lui rendent hommage en 1932

45 Pierre Renouvin, «L'opinion publique et la guerre en 1917», Revue d'Histoire Moderne et Contemporaine, 15, 1968, pp. 4-23 ; Jean-Baptiste Duroselle, La Grande guerre des français. L'incompréhensible, Paris, Perrin, 1994, pp. 187-312 ; George-Henri Soutou, op. cit. 1989 ; Jean Jacques Becker et Serge Berstein, op. cit., 1990, p. 88 ; Jean-Lovis Robert, Les ouvriers, la patrie et la révolution: Paris 1914-1919, Besançon, Presses Universitaires de Franche-Comté, 1995. 
ce faire une partie des ressources économiques au détriment du ravitaillement des populations et des besoins de guerre. C'était là d'ailleurs pour bon nombre d'industriels une des rares justifications acceptables de l'interventionnisme d'État ${ }^{46}$. La pénurie croissante de transports maritimes et de matières premières avait entraîné en mars 1917 l'interdiction générale des importations, la réquisition de la marine marchande, la centralisation de l'achat et de la répartition des rares ressources. Considérant l'organisation française comme proche de l'anarchie, les alliés menaçaient d'interrompre leurs livraisons, exigeaient la centralisation des décisions et de l'action administrative, la gestion rationnelle des importations. Pour chaque type de produit, les alliés imposaient de disposer dans les transactions commerciales d'un seul interlocuteur. C'est l'origine de la création et de la politique généralisée des consortiums, des groupements de commerçants et de fabricants d'une même spécialité qui achetaient à l'étranger les matières premières et les revendaient à leurs membres sous le contrôle de l'État. Leur vocation est d'équilibrer et de sécuriser produits et profits des industriels. Le premier consortium ainsi mis en place est celui dédié aux importations de coton ${ }^{47}$. Le rationnement est quasiment généralisé. Toute la flotte marchande est en décembre 1917 placée sous le contrôle d'Étienne Clémentel, un pouvoir de coercition redoutable qu'il peut avoir à exercer auprès d'industriels récalcitrants. C'est donc dans un contexte de crise et d'un Parlement docile qu'Étienne Clémentel pense aller plus loin dans la voie d'une renégociation des relations entre l'administration ef les agents de l'économie.

La liberté de fait laissée auxindustriels dansl'urgence et l'absence de contrôles dans ces circonstances avait donné lieu à d'inévitables fraudes, à la livraison de produits de mauvaise qualité et aux résultats approximatifs. D'où la nécessité et la justification de mobiliser les techniciens et les ingénieurs en mesure de contrôler, de juger, d'expertiser les méthodes de fabrication et leurs résultats en termes de produits finis. Albert Thomas avait dans cette perspective essayé d'imposer son autorité sur les industriels, mais sans succès ${ }^{48}$. II fallait donc bien négocier.

46 François Bouloc, Les profiteurs de guerre, 1914-1918, Paris, éd. Complexe, 2008.

47 " Consortium ou acheteur unique ", Archives nationales, F/12/7657.

48 Maxime Leroy, l'auteur en 1918 de Pour Gouverner, tient à jour des cahiers manuscrits dans lesquels il note des avis très critiques sur Albert Thomas. II lui reproche d'être « entouré de normaliens, innocentes âmes au milieu des pires audaces industrielles, [...] mal au
Étienne Clémentel s'entoure d'experts techniques et de scientifiques. II sait que pour agir efficacement il lui faut emporter l'adhésion des industriels et des agents de la production, et pour cela disposer non seulement d'une autorité légale, mais aussi d'arguments solides en vue d'une objectivation de la décision et que ses personnels sont chargés de lui fournir. Il a besoin de la caution d'experts en mesure de répondre rapidement à des questions simples. La valeur réelle de la réponse n'était peut être pas le plus important, mais une capacité de réaction et l'assurance de disposer en toute circonstance d'une solution contribuent à légitimer les initiatives. D'où l'impératif d'une équipe de spécialistes toujours plus étoffée faisant autorité dans tous les domaines. Ils devaient disposer de compétences, de connaissances, d'une analyse la plus proche possible de la situation réelle en vue de planifier l'action avec l'ensemble des agents économiques. Pour compléter la panoplie de ses services techniques et comités d'experts, Étienne Clémentel avait besoin d'une véritable armée d'enquêteurs lui permettant de l'informer dans cette perspective d'objectivation des analyses, des fins et des moyens à mettre en œuvre.

L'équipe des experts enquêteurs et rédacteurs Léon Guillet remplit les fonctions de rapporteur général du comité des études du Comité Consultatif des arts et manufactures à l'après-guerre ${ }^{49}$. C'est lui qui est chargé en 1917 de constituer l'équipe des enquêteurs et des rédacteurs, mais aussi d'assurer la coordination des travaux en lien direct avec le Ministre. La part belle est octroyée à la direction technique. C'est à elle qu'il revient de formuler en dernière instance les avis, de retenir ce qui doit l'être permettant la prise de décision : une façon de dire la supériorité de l'expertise technique sur la politique.

Les principaux responsables des comités ou des commissions d'études sont des scientifiques ou des ingénieurs. Outre Léon Guillet lui-même, on compte parmi eux le président du Comité Consultatif des arts et manufactures Albin Haller, professeur à la Sorbonne et directeur de l'École Nationale de Chimie et de

courant du trafic d'influence qui se nove autour de lui $॥$ : Extraits des Cahiers noirs de Maxime Leroy, inédits, déposés à l'Institut d'histoire sociale, cités par Christophe Prochasson, Les intellectuels, le socialisme et la guerre 1900-1938, Paris, Seuil, 1993, p. 298 et 309. 49 Lettre du Ministère du commerce à Léon Guillet, le 2 octobre 1918, F/12/7657 : « Arrêté du 28 septembre 1918 créant auprès du cabinet du ministre une direction des études techniques fixant les attributions de cette direction et vous chargeant des fonctions de directeur des études techniques $)$. 
Physique Industrielle. Le vice-président est Gabriel Chandeze, directeur honoraire du Conservatoire National des Arts et Métiers. L'autre vice-président est Henry Le Chatelier, maître de Guillet qui l'a coopté dans cette affaire ${ }^{50}$. II est par ailleurs un polytechnicien, professeur à la Sorbonne et le propagandiste zélé du taylorisme en France. Henry Le Chatelier, Gabriel Chandeze, Théophile Schloesing, Arsène d'Arsonval sont les présidents des quatre commissions techniques associées aux travaux de la Direction technique du ministère et du Comité Consultatif des arts et manufactures (respectivement "Métallurgie et construction mécanique "; "Textiles et papiers "; "Industries chimiques "; "Industries diverses »). Excepté Gabriel Chandeze, tous sont des académiciens.

La composition générale des comités eux-mêmes ne manque donc pas de légitimité tant scientifique que technique. Sur un total de 56 membres, huit sont académiciens; seize sont des chercheurs et professeurs de l'enseignement supérieur ; 25 sont sortis d'une école d'ingénieur et exercent dans l'industrie ou sont fonctionnaires d'un ministère technique ; neuf sont des industriels en activité, représentants syndicaux ou de chambre de commerce. Étienne Clémentel estime que dix d'entre eux doivent être avant tout considéré comme des savants ; 32 comme des industriels; et 14 comme des fonctionnaires. Le Comité Consultatif des arts et manufactures brille au moins par sa haute autorité scientifique et technique. De fait, ceux qui assurent la part de loin la plus active au sein de ces instances sont les savants et les ingénieurs. Ce sont eux qui coordonnent, organisent les études, sont les rapporteurs en dernière instance des documents (officiels et non officiels) que l'on retrouve dans les archives.

Le ministère du commerce devenait le centre d'élaboration des plans économiques pour l'après-guerre. Le rapport de 1919 se devait de porter un projet et une ambition politique : assurer l'avènement administratif des producteurs dans le cadre d'une rationalisation de l'organisation de la production industrielle sous l'égide des experts. II s'agissait d'impulser désormais une transformation radicale de la société française devant lui permettre d'assurer son expansion économique face aux nations rivales, et en particulier l'Allemagne. Ce qu'Étienne Clémentel ne conçoit plus que sur la base d'une action unanime devant aboutir à la rationalisation de l'ensemble des activités sociales et

50 Michel Letté, Henry le Chatelier (1850-1936) ou la science appliquée à l'industrie, Rennes, Presses universitaires de Rennes, 2004. économiques. II revenait à ses services techniques, ses experts et scientifiques de déterminer les moyens appropriés pour conduire ce dessein.

\section{Un manifeste pour rationaliser l'économie}

La quête d'une troisième voie

Ce rapport n'est en aucun cas la traduction d'une volonté d'étatisation de l'économie qui se serait reniée une fois évanovies les contraintes de guerre. Étienne Clémentel n'y a en réalité jamais songé au motif que la guerre l'imposait. Au contraire. L'état d'esprit qui préside à l'élaboration de son programme est de se prémunir d'une remise en cause trop radicale de l'ordre économique libéral d'avant-guerre. Certes il juge comme une évidence de devoir administrer, orienter, encadrer l'économie, mais certainement pas de condamner son régime antérieur au profit d'une emprise totalisante de l'État. Sa proposition doit plutôt être considérée comme la crainte de ce qu'Étienne Clémentel considère comme une véritable menace pour la démocratie industrielle : celle du collectivisme où l'État voit ses prérogatives s'élargir sans limites. Le programme suggéré vise en fait à échapper à l'alternative du libéralisme traditionnel et du socialisme, tout en veillant à préserver l'ordre libéral et ses valeurs, à pérenniser un système social et économique d'avant-guerre qu'Étienne Clémentel défend sur le plan intellectuel et politique. Premier auteur mobilisé dans son rapport, Adam Smith est là pour rappeler ses idéaux. Comme les penseurs classiques et leurs épigones qui reformulent la théorie économique libérale dans les années 1870, Étienne Clémentel prétend formuler des lois quasi naturelles en matière d'économie industrielle. II revendique la légitimité d'un régime libéral organisé comme le seul capable d'assurer la prospérité du pays, à condition toutefois de l'aménager et d'agir sur ce qu'il considère comme les travers français de la production industrielle et de son commerce, de confier aux producteurs et aux élites dirigeantes la conduite des initiatives collectives.

Le rapport d'Étienne Clémentel doit donc être aussi lu comme la quête d'une évolution de l'économie libérale d'avant guerre vers une voie qui l'éloigne du spectre socialiste. II faut en analyser les contenus idéologiques et leurs valeurs. Ce n'est ni le socialisme que propose Étienne Clémentel, ni le libéralisme économique orthodoxe, mais bien la mise au travail, la mise en conformité de l'organisation et du gouvernement rationnel des agents économiques avec les impératifs du productivisme. La rationalisation comme instrument d'une domination des élites a dans ce cas pour fonction d'opposer 
au collectivisme révolutionnaire et au socialisme un contre-modèle faisant de l'intérêt collectif opposé à l'individualisme l'objectif de sa politique. Le " collectivisme rationnel » permet selon lui de canaliser les prétentions révolutionnaires au travers de la quête d'une politique conciliatrice entre les tenants de la conservation libérale, de la réforme sociale et les aspirations socialistes de la classe ouvrière. Ce qui se met en place durant la guerre s'inscrit dès lors dans la continuité de la tradition élitiste de la IIle République, avec pour horizon l'objectivation des modes d'organisation et d'administration par l'État d'une économie orientée par les experts et la mise en œuvre technique par les agents producteurs.

\section{Le centralisme technocratique}

Les injonctions à la rationalisation de l'économie invitent dès lors à l'instauration d'un régime gestionnaire et technocratique des affaires industrielles et commerciales. Elles recommandent un nouvel ordre social saisi par l'intégration des travailleurs et la promesse de profits du fait d'une croissance inéluctable des richesses, mais aussi par la promotion d'une culture de la science et de la technique où les savoirs permettent la prise de décision rationnelle et non négociable. Cette revendication d'une économie s'érigeant sur la neutralité de savoirs objectifs permettrait ainsi, en la technicisant, d'échapper en partie à la décision politique et à la connaissance collective. Ainsi le projet pérennise les principes traditionnels d'une III République autoritaire où l'efficace, la performance et la dynamique de perfectionnements permanents justifient la prise en charge par les élites techniques et expertes des orientations de l'organisation économique et de la réforme sociale ${ }^{51}$.

De ce point de vue, le rapport d'Étienne Clémentel peut être considéré comme la manifestation d'une emprise technocratique sur la politique économique et social au sortir de la guerre, un matériau pour l'histoire de ce qui sera un peu plus tard désigné par « capitalisme d'État» ou "économie administrée ". "l est aussi un matériau pour l'histoire de ces élites qui ont en charge la conduite de l'économie dans les années 1920 : les industriels toujours, mais surtout les technocrates, les experts, et les scientifiques qui trouvent l'opportunité de consolider des liens novés avantguerre sur la base d'une montée en rationalité de

51 Gérard Brun, Technocrates et technocratie en France (1914-1945), Paris, Éditions Albatros, 1985 ; Dominique Janicaud, La puissance du rationnel, Paris, Gallimard, 1985. l'organisation de la société industrielle ${ }^{52}$. C'est ce que John Godfrey suggère avec la démonstration qu'Étienne Clémentel a été l'artisan principal de la construction administrative de l'intervention étatique, notamment à partir de l'organisation des consortiums destinés à réguler et contrôler la circulation des marchandises ${ }^{53}$. II analyse les mécanismes de la politique conduite sous l'égide de ce ministère de l'économie rationnelle, mais aussi les principes du "capitalisme organisé " et du "corporatisme $\|^{54}$. C'est également la thèse d'Adrian Rossiter. II situe pour sa part la politique et l'action d'Étienne Clémentel dans une perspective plus large, celle de la construction dans l'entredeux guerres de systèmes de direction et de management de l'économie sous la houlette du corporatisme d'État et de la technocratie légitimant ses options par l'apolitisme des experts et de leurs avis. II défend de façon convaincante que c'est là un registre essentiel de la stabilité de la IIle République à laquelle Étienne Clémentel a indéniablement contribué par son action gouvernementale ${ }^{55}$. Au demeurant, les termes " rationalisation " et " néo-capitalisme " sont, dans ces deux derniers textes, pour ainsi dire synonymes.

\section{Conclusion}

La guerre avait autorisé la réalisation consensuelle de l'utopie technocratique d'une gestion de l'économie et du social où l'adhésion volontaire à l'autorité de la science et de la technique détermine des choix politiques, guide le comportement rationnel

52 Aimée Moutet, Les logiques de l'entreprise. La rationalisation dans l'industrie française de l'entre-deux-guerres, Paris, Ehess, 1997 ; George G. Humphreys, Taylorism in France, 1904-1920: the impact of scientific management on factory relations and society, New York, London, Garland, 1986 ; Patrick Fridenson, « Un tournant taylorien de la société française (1904-1918) 1, Annales ESC, 42 (5), 1987, pp. 10311060 ; Charles S. Maier, « Entre le taylorisme et la technocratie : idéologies et conceptions de la productivité industrielle dans l'Europe des années 1920 "), dans Lion Murard et Patrick Zylberman (dir.) Le Soldat du travail : guerre, fascisme et taylorisme, Recherches 32/33, sept. 1978, pp. 95-134 ; Lyndall F. Urwick, "The rationalization movement ", dans Leslie Hannah (ed.), The rise of the corporate economy, London, J.H. University Press, 1976, pp. 29-44 et The Meaning of Rationalisation, London, Nisbet, 1929.

53 John F. Godfrey, op. cit., 1987, pp. 82-180.

54 Charles S. Maier, Recasting Bourgeois Europe : Stabilization in France, Germany, and Italy in the Decade after World War I, Princeton New Jersey, Princeton University Press, 1975.

55 Adrian Rossiter, Experiments with corporatist politics in republican France, 1916-1939, Oxford, Thesis Nuffield College, 1986. 
des agents économiques. Étienne Clémentel s'est fait à cette occasion l'avocat, peut être non de la technocratie elle-même, ou même de l'économie dirigée, mais au moins - comme il le dit lui-même de l'économie "orientée par l'État ॥. \| apparait pourtant bien tel un chef de file parmi les élites républicaines d'obédience technocratique qui s'affairent à stabiliser l'ordre social par la promotion de la rationalisation, ou du moins en en créant les conditions. Nul doute que la guerre et sa promesse d'une conclusion victorieuse au prix d'une réorganisation rationnelle de la société industrielle et de sa délégation aux technocrates auront contribué à édifier, avec Étienne Clémentel, la perspective d'un avenir suffisamment optimiste et crédible pour déclencher les actions qui engendreraient sa propre réalisation. L'idée s'est répandue d'une nécessaire délégation à l'État et à ses scientifiques, à ses techniciens qui acquièrent désormais toute légitimité pour prévoir, organiser et agir en matière d'économie sous l'égide de la rationalisation.

Étienne Clémentel aura créé pour les compétences techniques et les experts l'opportunité d'une consolidation de leurs rôles au sein des instances gouvernementales, mais aussi parmi les commissions, les comités et les offices dédiés qui consolident ainsi l'autonomie de l'administration par rapport au gouvernement ${ }^{56}$. C'est en tout cas ce que les technocrates dans le giron d'Étienne Clémentel cultivent et tentent de prolonger en invoquant la nécessité de poursuivre en temps de paix, et sur le modèle des actions engagées lors du conflit, la rationalisation de l'État et de ses pratiques en matière d'économie. Ce vœu d'institutionnaliser leur fonction politique s'exprime clairement dans le rapport de 1919, notamment par la proposition d'instaurer un Conseil d'État Technique sans lequel les parlementaires et le législateur ne pourraient arrêter de décision. Constitué d'experts scientifiques et de techniciens, cette instance serait l'une des bases du dirigisme technocratique tendant vers la rationalisation de la politique. Ce conseil voit partiellement le jour cing ans plus tard. Le scientifique Paul Painlevé inaugure en 1924 le Conseil économique national, mais tout en ignorant les aspects les plus technocratiques qui s'étaient exprimés sans complexe dans le rapport d'Étienne Clémentel| ${ }^{57}$.

56 Francine Soubiran-Payet, "Parlement, administrateurs et experts (1900-1914). Le discours de la compétence ", Vingtième Siècle, 93, 2007, pp. 151-163; Alain Chatriot, "Les offices en France sous la Troisième République. Une réforme incertaine de l'administration $»$, Revue française d' administration publique, 120, 2006, pp. 635-650.

57 Adrian Rossiter, op. cit., 1987 ; Alain Chatriot, La démocratie
En définitive, l'équipe des enquêteurs, avec leurs travaux et leurs réflexions restitués ici sous la forme de ce document ont constitué une sorte de laboratoire de la technocratie politique et sociale. Ils ont fonctionné comme l'un de ces nombreux espaces du débat extraparlementaire, ou encore comme l'une de ces annexes de la Chambre qui se sont multipliées depuis la fin du siècle autour des questions de la réforme sociale ${ }^{58}$. Cette histoire se prolonge assurément au-delà du rapport Clémentel, la rationalisation devenant dans l'entredeux guerres contagieuse. D'autres cadres de pensée s'affirment, permettant au monopole de la rationalité instrumentale de s'exercer sur la raison, à la rationalisation de devenir l'apanage de la société industrielle et du gouvernement des agents économiques comme des populations. sociale à la française. L'expérience du Conseil national économique, 1924-1940, Paris, La Découverte, 2002.

58 Alain Chatriot, "Réformer le social sous la Troisième République ", Revue d'histoire moderne et contemporaine, 56 (suppl.), 2009, pp. 40-53. 
Retranscription des pages I à XXVI, d'après le volume 1 du Rapport général sur l'industrie française, sa situation, son avenir (1919)

\section{MONSIEUR LE PRÉSIDENT,}

J'ai l'honneur de vous présenter le Rapport général sur l'Organisation de la Production nationale au lendemain de la guerre, résultat des travaux du Comité consultatif des arts et manufactures et des enquêtes de la Direction des Études techniques du Ministère du commerce.

Afin de diriger les techniciens du Comité consultatif des arts et manufactures et mes collaborateurs dans leurs études et de coordonner leurs efforts, je leur ai demandé, en présidant la séance d'inauguration de leurs travaux, de s'inspirer des principes suivants dont l'application est la condition même du relèvement économique de notre pays éprouvé par quatre années de luttes sanglantes et dévastations systématiques : accroître dans toute la mesure du possible notre productivité ; réduire nos importations en utilisant à leur plein rendement nos ressources nationales, métropolitaines et coloniales; développer au maximum nos exportations. Ces principes, posés au frontispice de l'œuvre, en 1917, je les retrouve aujourd'hui, sous forme de conclusion, confirmés par l'enquête générale sur la situation de notre industrie avant, pendant et après la guerre.

Je crois devoir tracer ici dans leur grandes lignes les résultats des travaux que viennent de mener à bonne fin mes collaborateurs et qui forment un corps complet de doctrine économique s'appuyant sur une abondante documentation, recueillie et mise en œuvre par $M$. Léon Guillet, Directeur des Études techniques. Ce corps de doctrine forme en réalité un grand programme de l'œuvre à accomplir dans toutes les branches de l'activité industrielle nationale. Il restera la directive générale de l'œuvre gigantesque qui s'offre aux Décisions du Gouvernement et du Parlement et à l'activité corporative du monde de la production.

Pour élever nos ressources au niveau de nos propres besoins, pour nous libérer de notre dette à l'extérieur, nous devons accroître notre richesse nationale. Or, pour les nations comme pour les individus, il n'est qu'un moyen honorable et efficace de devenir plus riche, c'est de travailler davantage, plus vite et mieux, c'est d'intensifier la production. Mais la production est fonction des débouchés et on n'étend ses débouchés qu'en abaissant ses prix de vente, donc ses prix de revient. A cette seule condition, nous augmenterons notre capacité productive.

La production exige d'abord des producteurs. Dans notre pays de faible natalité, le capital humain, de tous le plus précieux, si précieux qu'il faut renoncer à matérialiser sa valeur en chiffres, a subi une déperdition effroyable. Des sept millions d'hommes successivement mobilisés, deux millions, morts, infirmes ou mutilés, doivent malheureusement figurer au bilan des pertes ou des non-valeurs de la production nationale. Et ce sont souvent les meilleurs qui se sont sacrifiés, les meilleurs moralement et économiquement, les meilleurs par la vigueur du caractère et la force de l'âge.

En vue de combler ce vide, on a pensé à recourir à la main d'œuvre étrangère; cette solution simpliste ne serait pas sans dangers pour l'intégrité de notre race et le maintien du niveau de vie du travailleur national; elle ne saurait intervenir que dans des circonstances déterminées, avec des précautions préalables. Nous possédons chez nous des ressources latentes dans une meilleure distribution des activités économiques. Je ne fais pas allusion à la main d'ouvre féminine; car il y a des inconvénients d'ordre social à aller trop loin dans une voie où des prodiges d'énergie ont déjà été accomplis. Mais, par le jeu même des forces économiques, toute action par voie de contrainte étant inopérante en ces matières, on peut concevoir un meilleur classement des professions, un passage des professions relativement improductives aux professions productives. Sous l'influence immédiate de la guerre, nous 
avons constaté des déplacements de cet ordre : petits rentiers contraints au travail par la vie chère, domestiques appelés à l'usine par les hauts salaires, etc.

Bien inutilement, nous aménagerions au mieux nos ressources actuelles si elles devaient diminuer progressivement $d u$ fait du fléchissement inquiétant de notre natalité. L'économiste ne peut rester insensible aux conséquences tragiques de cette crise de la vitalité française ; il étudie passionnément les réformes proposées pour conjurer le péril ; il place sa confiance dans le besoin instinctif de l'humanité de croître et de multiplier au sortir du cycle de la mort. Jusqu'au jour où, des berceaux maintenant vides, sortiront plus nombreux les travailleurs de l'avenir, nous devons veiller à ce que les tombes ne soient pas prématurément ouvertes par des maladies évitables; nous continuerons surtout à combattre les fléaux sociaux que sont la tuberculose, la syphilis et l'alcoolisme.

Fort heureusement, sans attendre la réalisation de ces lointains espoirs et le résultat de ces mesures, nous pouvons immédiatement nous procurer une main d'œuvre plus abondante : la machine libère du travail humain, disponible pour des auvres nouvelles, et elle augmente le rendement du travail retenu, devenu plus facile et plus efficace. Or, dans notre France, la première à faire surgir les inventions, la dernière trop souvent à les réaliser, il existe peu d'industries dans lesquelles le perfectionnement de l'outillage ne puisse conduire à une production supérieure. Mais les nécessités de l'évolution industrielle triompheront de la routine. Le machinisme pénétrera au domicile de l'artisan et étendra son action dans l'usine la plus modeste.

Dans la grande industrie, on atteindra des résultats qui transformeront entièrement les conditions de la production. Ainsi, de plus en plus, l'intelligence de l'inventeur, incorporée dans les organes de la machine, substituera au dur labeur musculaire le simple effort d'attention dont se contente un rôle de contrôle et de direction.

Les résultats escomptés des progrès de l'outillage seraient rapidement compromis si l'amélioration physique, intellectuelle, morale et technique du travailleur ne marchait pas de front, si le capital vivant ne se montrait pas supérieur au capital inerte qu'il doit vivifier. Mais on ne saurait accepter cette hypothèse à l'heure où nous sommes et dans notre pays. C'est la raison d'être de la Démocratie de tendre au développement de toutes les facultés, de tous les individus, sans distinction; en se réalisant sur le terrain du social, elle nous donne des corps plus vigoureux, des intelligences plus ouvertes, des consciences plus droites; en se réalisant sur le terrain économique, elle rend l'ouvrier plus apte par l'apprentissage et l'enseignement technique à l'exercice de son métier. Nous sommes donc en droit d'espérer que, dans l'usine modernisée, les agents d'exécution seront capables d'obtenir un plein rendement du machinisme le plus perfectionné, créé par nos élites scientifiques et installé par des chefs d'entreprise audacieux et novateurs.

Les qualités des producteurs acquises et confirmées, il faut les utiliser au mieux par une distribution rationnelle des fonctions individuelles dans l'œuvre collective. Ici intervient l'organisation du travail, basée sur l'étude expérimentale des mouvements dans le corps humain en pleine action professionnelle et l'exacte coordination des rôles assignés à chaque exécutant. L'idée maîtresse est de n'imposer à aucun ouvrier, par des mouvements superflus, une fatigue inutile, et de faire passer les mouvements nécessaires à l'état d'habitude; il en résulte un effort moins grand pour les muscles, moins de tensions pour le cerveau. L'effort de réflexion est fait à l'origine par les ingénieurs auxquels incombent les dispositions préparatoires; dans le concert ainsi réglé à l'avance et qui n'admet point la moindre défaillance, le manœuvre d'antan vient remplir son rôle en collaborateur souple et intelligent. Abandonnée à l'empirisme, la division du travail était déjà l'agent par excellence de la production intense; le premier, Adam Smith en célébra la puissance féconde; transformée par l'expérimentation scientifique, elle développera toutes les vertus qui sont en elle et produira des merveilles insoupçonnées. Les résultats acquis sont concluants : les plus-values 
de production vont du triple au décuple, surtout dans le compartiment des constructions mécaniques, qui est précisément celui pour lequel nous comptons le plus étendre nos exportations. Bien entendu, la formule du maximum de travail dans le minimum de temps doit se compléter par celle du maximum de salaire dans le maximum d'hygiène et de bien-être.

L'organisation méthodique du travail est sous la dépendance de l'uniformité des types, c'est-à-dire de la standardisation qui, seule permet le travail en série, au premier degré et la spécialisation des usines au deuxième degré. Les rapports du Comité consultatif exposent les conditions auxquelles chacune de nos diverses industries pourra adapter ces méthodes décisives de rendement intensif. Dans cet ordre d'idées, j'ai créé la Commission permanente de Standardisation et la Commission d'Unification des Cahiers des Charges. C'est surtout dans ce domaine que nous étions dépassés par nos rivaux. Or la guerre a développé fatalement la grande industrie, celle où une fabrication toute mécanique jette sur un large marché une masse de produits semblables. Dans les luttes pacifiques, comme dans les luttes guerrières, l'action de masse est devenue prépondérante. Tout en nous pliant à la satisfaction des besoins les plus vulgaires qui, communs à tous, fournissent les débouchés les plus étendus, nous saurons conserver notre supériorité antérieure dans les industries de luxe qui, confinant à l'art et visant l'élégance, répandent sur le monde l'influence du goût français.

L'État contribuera très efficacement à ces progrès d'ordre technique par les laboratoires et les instituts que nous nous sommes attachés à créer et à développer : le savant doit sortir de sa tour d'ivoire et l'industriel s'élever au-dessus des préoccupations immédiatement utilitaires; la théorie qui ne passe jamais à la réalisation nous est indifférente, et la réalité qui n'est pas animée par l'esprit de progrès est bientôt frappée de stérilité. Les nécessités de la guerre ont mis en contact le savant et l'industriel; désormais ils ne s'ignoreront plus.

Intelligences, énergies, compétences, tout sera frappé de paralysie si nous ne disposons pas des moyens financiers qu'exige l'exécution d'un vaste programme de réorganisation économique. Pour les industries ruinées à reconstituer, pour les industries nouvelles à créer, pour les industries archaïques à moderniser, pour l'outillage économique à développer, les capitaux seront sollicités dans des limites inconnues jusqu'à présent. Ces capitaux, ce n'est pas uniquement à un État endetté par la guerre que l'on peut songer à les demander : c'est aux organes normaux du crédit, c'est aux banques. Mais elles ne pourront remplir ce rôle que si, instruites par les résultats néfastes de l'expérience d'avant-guerre, elles consentent à devenir les collaboratrices de nos nationaux. Sur le chiffre des valeurs émises avant 1914, plus de la moitié allait fructifier à l'étranger. Nous nous comportions comme un industriel qui, ayant des capitaux disponibles, les offriraient à un concurrent et laisserait, faute de ressources, péricliter sa propre entreprise. Par une étrange contradiction, certains grands établissements s'interdisaient tout emploi de fond qui les aurait immobilisés dans l'industrie en France, même avec un bénéfice certain et appréciable, tandis que dans les placements à l'étranger, leur confiance et leur hardiesse ne connaissaient souvent pas de bornes.

La France, par les vertus patientes de son épargne, était devenue un réservoir mondial de capitaux; mais, par la suite d'une déplorable éducation financière, ces capitaux, elle ne savait pas les rendre productifs chez elle. Bourgeois et paysans recherchaient surtout les placements dits "de père de famille », à revenus limités, sans se dire que les revenus dont le maximum est fixé ne sont cependant pas des revenus garantis contre tout risque et oubliant que, dans ce cas, le prêteur, qui n'a aucune chance de majoration, a contre lui toutes les chances de perte. En regard du capitalisme anglais et du capitalisme allemand, foncièrement dynamiques, le capitalisme français, timide et conservateur, paraissait devoir rester incurablement statique.

Toutefois, depuis quelques années, nous avons vu se manifester les symptômes d'un renouveau financier. On aurait pu croire que toute activité bancaire régionale serait rendue impossible en province. Heureusement, les meilleures banques locales survécurent à la crise 
de concurrence à tendances centralisatrices qu'elles durent subir et en sortirent plus vigoureuses. Elles ont trouvé dans l'union et la fédération les moyens de résister et de vaincre. Depuis vingt ans, nos banques régionales ont réalisé le rapprochement des industriels et des banquiers; elles ont collaboré puissamment au merveilleux développement économique de la Flandre, de la Lorraine et du Dauphiné. Elles sont prêtes à jouer un rôle prépondérant dans l'œuvre de restauration et d'expansion qui suivra la guerre.

Voici donc nos capitaines d'industries entourés d'une incomparable armée du travail, à même de faire fructifier les capitaux lentement constitués par l'épargne française. Des richesses, latentes dans notre sol, s'offrent à eux. Mais un problème d'une particulière acuité se pose dans toutes les branches de leur activité. Où puiser l'énergie, la force motrice? Dans l'établissement des prix de revient, c'est toujours là que l'on rencontre l'obstacle le plus difficile à franchir. Une différence de quelques francs par tonnes dans le prix du combustible met nos produits manufacturés dans une position d'infériorité à l'égard de ceux de nos concurrents qui peuvent se procurer le charbon à meilleur marché et en quantité suffisante. Notre production était inférieure de 20 à 25 millions de tonnes à nos besoins avant la guerre, elle le sera davantage après la guerre par la suite des destructions de l'ennemi et des besoins de l'Alsace-Lorraine.

Pour combler ce déficit, nous devons pouvoir compter tout d'abord, et pour une très large part, sur les restitutions en nature que l'Allemagne devra nous fournir. Le charbon allemand, dont le prix de revient doit être tout au plus égal au prix de vente du charbon anglais en Angleterre, nous assurera des quantités importantes à des prix favorables.

Mais nous devons, d'autre part, faire les plus grands efforts pour parer par nous-mêmes, dans la plus grande mesure possible, à notre déficit.

Nous pouvons tout d'abord réaliser des économies considérables dans la consommation. Parmi les procédés à employer à cet effet, on envisage la distribution de la force par de grandes stations électriques où sont brûlés des charbons de seconde qualité, envoyés trop souvent aux déblais, ou les lignites insuffisamment exploités dans notre pays qui alimentaient les formidables stations allemandes.

On préconise des mesures rendant obligatoires l'utilisation du gaz des hauts fourneaux et la débenzolisation du gaz d'éclairage; on étudie également un meilleur emploi du combustible par le perfectionnement des appareils de chauffage. Une économie de houille résultera enfin de la multiplication des moteurs à explosion qui, il est vrai, actuellement, consomment surtout un carburant étranger, mais qui trouveront le moyen de remplacer le pétrole, soit par l'alcool, soit par le benzol, soit par les huiles provenant de la distillation des schistes et autres produits aujourd'hui incomplètement utilisés.

Toutes ces mesures donneront, certes, des résultats. La houille blanche offre une solution plus large; non seulement elle se substitue à la houille noire, mais encore elle accomplit des merveilles auxquelles ne peut prétendre sa rivale; nos industries chimiques et métallurgiques, renouvelées par elle, marcheront au premier rang dans la voie du progrès. De nos forces hydrauliques, nous tirons à l'heure actuelle, grâce à l'effort de guerre, environ 1 million de chevaux : il en reste 7 millions disponibles qui doivent entrer en activité sans tarder. Leur aménagement se prépare avec activité. Nous avons donc la certitude d'améliorer considérablement les conditions de production de l'énergie souveraine qui commande toutes les transformations industrielles.

Pour en tirer tout le profit que nous pouvons espérer, nous devons songer à diminuer le coût de la vie qui, lui aussi, pèse lourdement sur les prix de revient, en intensifiant la production des principales denrées alimentaires. Assurément, c'est ici le domaine réservé de l'agriculture, mais, sans parler de son industrialisation croissante, il faut noter que la production agricole ne suffira à nos besoins qu'en s'appuyant sur une grande industrie 
chimique qui lui donnera les engrais azotés, phosphatés et potassiques. En 1880, le rendement par hectare de la culture du blé était, en France, comme en Allemagne, de 11 quintaux. Mais, grâce à un emploi approprié des engrais chimiques, ce rendement en 1913 passait en Allemagne à 20 quintaux tandis qu'en France il s'élevait péniblement à 13,52. Le Danemark a connu des rendements de 33 quintaux. Les nécessités de la guerre ont provoqué la création d'usines capables de nous procurer, sur notre sol, une partie importante des nitrates dont nous avons besoin ; grâce à nos admirables gisements du Nord de l'Afrique, nous sommes mieux placés que quiconque pour intensifier la production des superphosphates. Un engrais essentiel nous manquait pour rajeunir la vieille terre de France, la potasse. Nous la possédons en surabondance depuis la désannexion de l'Alsace. Aux directeurs de nos exploitations agricoles maintenant d'appliquer ces moyens puissants à la régénération du sol, en vue d'obtenir les 20 ou 25 quintaux par hectare qui nous permettront, soit de devenir exportateurs de céréales, soit de libérer de la culture du blé un million d'hectares qui pourront être consacrés à l'élevage ou aux cultures industrielles.

Tirer nos subsistances de notre propre fonds est une condition de sécurité aux heures de perturbation mondiale; les obtenir à des prix modérés est une garantie permanente contre l'élévation du prix de la vie, qui handicaperait notre production et contribuerait à rendre pénible et précaire la condition du travailleur.

Mais subvenir à la consommation des masses laborieuses, ce n'est pas encore faire auvre positive de production. Celle-ci commence avec le ravitaillement en matières premières de nos industries de transformation. Certes, nous possédons sur notre propre sol certaines matières premières parmi les plus importantes, mais il en est pour lesquelles nous sommes tributaires de l'étranger. Ne nous dissimulons pas que, pour ces dernières, nous aurons gravement à souffrir du déséquilibre économique créé par la guerre; les stocks actuels ne répondent pas à des besoins impatients d'être satisfaits. La concurrence mondiale déchaînée entraînera sur certains produits une hausse factice des prix qui pèsera lourdement sur la France momentanément désavantagée par des conditions particulièrement difficiles de ses moyens de transports maritimes et de ses moyens de payement internationaux. Nous devons cette situation au redoutable honneur d'avoir été, dans le temps, l'avant-garde de la Ligne des Nations, luttant pour la civilisation, et, dans l'espace, le théâtre des batailles gigantesques accumulant les ruines et les dévastations. C'est pourquoi, nous nous étions toujours préoccupés, au cours de la guerre, de faire sortir la France de son isolement par un appel à l'équité de nos associés, et de lui procurer le bénéfice du fonctionnement des organisations interalliés grâce auxquelles elle a pu vivre et vaincre. Au lendemain de l'armistice, les organismes, n'étant plus maintenus par la pression de la guerre dans leur discipline étroite, se sont peu à peu détendus, chaque pays revenant progressivement à ses propres responsabilités et le monde, peut-être trop rapidement, à une liberté économique générale.

Cependant, espérons que, devant les nécessités de la situation, l'œuvre de coopération entre les Alliés survivra à la Paix qu'ensemble ils ont si chèrement conquise. Espérons notamment que nos grands Alliés comprendront que, pour réédifier le monde sur des bases solides, ils ont le devoir d'aider les peuples qui ont le plus souffert de la guerre, et au premier rang la France et la Belgique, à régulariser et à stabiliser leur situation financière et à améliorer leur change. Le monde reprendra sa vie saine et normale d'autant plus vite que le problème financier sera résolu plus rapidement grâce à des mesures communes.

Cependant, malgré notre espoir, agissons comme si nous devons compter que sur nousmêmes. Le sentiment est peut-être un facteur important des rapports politiques internationaux; les rapports économiques sont malheureusement sous la seule dépendance des intérêts vitaux et permanents. Et, quand bien même le sentiment y jouerait un rôle, il ne 
faut pas oublier que, dans la reconnaissance, ce n'est pas l'élan premier mais la durée qui est le point difficile.

Préparons-nous à développer toutes nos ressources. Quand la période de crise consécutive à la guerre aura pris fin, nous nous féliciterons d'avoir édifié une économie nationale harmonieuse. Nous pouvons trouver sur notre territoire métropolitain et dans notre empire colonial qui s'étend sous toutes les latitudes, donc sous tous les climats, la plupart des matières premières et des denrées alimentaires que nous demandons actuellement à l'étranger.

Nos industriels du coton sont tributaires des États-Unis, de l'Inde et de l'Égypte. Il ne tient qu'à eux de conquérir leur indépendance. Dans les vallées du Sénégal et du Niger, d'immenses terrains d'alluvions n'attendent, pour être mis en valeur, que l'effort méthodique des ingénieurs et des capitalistes. Depuis plusieurs années, le Gouvernement général de l'A.O.F. fait procéder aux études préparatoires de tout un ensemble de travaux d'irrigation. Quand le groupement des industriels qui transforment le coton aura compris qu'il doit aider les organisations capables de produire cette précieuse fibre sur le territoire national, l'avenir de l'industrie cotonnière en France pourra être envisagé avec sérénité.

$\mathrm{Si}$, pour cette matière première, nous sommes seulement en présence de mesures préparatoires, pour d'autres, comme les graines oléagineuses, nous sommes en présence de réalisations immédiates. En quelques années, nous pourrons porter la production au niveau de la consommation en la poussant par divers moyens : subventions aux syndicats agricoles, décortication sur place, construction de docks et entrepôts, aménagement des ports, création de laboratoires dirigés par des chimistes spécialisées. Pour ne citer qu'un exemple, au Sénégal, le long d'une voie ferrée à peine construite, on a vu le cultivateur noir semer l'arachide dans les terres fraîchement débroussaillées et alimenter le trafic des premiers wagons mis en circulation.

La marge entre la production coloniale et la consommation métropolitaine est malheureusement encore trop large pour la plupart des matières premières : minerais et métaux, peaux, laines, bois, caoutchouc, cacao, etc. Mais nous pouvons affirmer, sans présomption, que cet écart ira rapidement en diminuant et qu'il arrivera un moment, si nous savons vouloir, où nous serons exportateurs, ce qui est déjà le cas pour la vanille, le riz, le poivre, la girofle et le raphia. M. le Ministre des Colonies vient de créer un service de la mise en valeur de nos richesses d'outre-mer qui stimulera, dirigera, coordonnera les efforts des divers Gouvernements coloniaux en vue du développement de la production. Il peut compter sur le concours le plus actif du Ministre du commerce pour la recherche des débouchés sur le marché national. L'organisation des groupements syndicaux rendra le contact facile et fécond entre colons ou indigènes et industriels de la métropole; ceux-ci doivent, en effet, considérer comme un devoir de placer les capitaux dans les entreprises coloniales qui les intéressent directement.

Déjà ce programme entre en action. C'est ainsi que vient de se fonder, sous l'impulsion du Ministre du commerce, l'" Office national des matières premières utilisées par la Pharmacie, la Distillerie et la Parfumerie». Cet office réalisera l'unité de direction des services dépendant à la fois des Ministères du Commerce, de l'Agriculture et des Colonies. Composé de savants, d'industriels, de professionnels des cultures spéciales de la métropole et des Colonies, il comprend une section de culture et de récolte des plantes indigènes, une section de culture et de production coloniale et une section d'importation des matières exotiques. Dès maintenant, il vient de prendre les mesures permettant d'assurer à la France l'approvisionnement, dans les délais imposés par un effort méthodique, de l'écorce de quinquina nécessaire au maintien et au développement d'une industrie née sur notre sol et dont le ravitaillement est aujourd'hui très précaire. 
Nos industriels, pourvus de l'énergie mécanique, des capitaux et des matières premières, nous les suivons, en parcourant les chapitres successifs de ce Rapport général, aux diverses étapes de la production. La compétence de nos ingénieurs et l'habilité de nos ouvriers, nous garantissent la qualité incomparable des marchandises lancées sur le marché mondial. A la base, c'est la métallurgie du fer, abondamment approvisionnée en minerai, capable de produire aussi bien les fontes vulgaires dans le haut fourneau colossal que les aciers les plus rares dans le four électrique. Au-dessus, viennent les industries de transformation de la fonte et de l'acier qui, en substituant l'exportation du produit fini à celle du produit brut, décupleront la valeur du même tonnage passant la frontière et feront pencher en notre faveur la balance du commerce extérieur. Ensuite, à l'étage supérieur, les industries mécaniques où excelle le génie français : machines-outils, machines agricoles, cycles, avions, automobiles, construction électriques, etc. Enfin, au dernier stade, les industries textiles qui n'ont qu'à redevenir ce qu'elles étaient avant les dévastations de l'ennemi pour constituer le gros chiffre de nos exportations, et les industries chimiques qui, transformées et galvanisées par la guerre, n'auront plus à redouter la concurrence allemande. En lisant ces monographies, dont chacune est solidaire d'un ensemble imposant, ou acquiert la conviction que l'industrie française peut aborder la lutte avec, pour elle, si elle sait vouloir, toutes les chances de la victoire.

Dans l'examen des procédés les plus propres à élever notre production, nous n'avons considéré jusqu'ici le chef d'entreprise que comme un être isolé, aménageant au mieux les valeurs humaines, les forces naturelles et financières qui concourent à la création des richesses. Il importe de s'élever à un degré supérieur d'organisation et de combiner les efforts de l'ensemble des procédures d'une même branche industrielle en vue de coordonner leur action tant sur le marché intérieur que sur le marché extérieur.

Dans la première phase d'individualisme effréné par laquelle a passé l'industrie, phase qui eut son utilité et sa grandeur, le producteur d'une marchandise quelconque était un loup pour le producteur de la même marchandise; l'écrasement d'un rival constituait un succès au même titre que l'ouverture d'un nouveau débouché; des ruines individuelles étaient la rançon des fortunes individuelles. La richesse nationale n'y trouvait pas toujours son compte. Trop souvent, en effet, la concurrence effrénée aboutissait à l'avilissement des prix et, par suite, à une crise dont souffraient même les vainqueurs, et dont le bien-être général était souvent le prix.

Depuis un quart de siècle, les producteurs avaient éprouvé le besoin de conclure des ententes, sortes de trêves entre les combattants qui se reconnaissent d'égale force, ententes ayant pour objet de substituer, par une réglementation volontaire, une production organisée à une production anarchique. A la faveur de la concentration industrielle et commerciale qui caractérise l'évolution économique moderne, on a constaté l'influence universelle d'un phénomène général qui, s'adaptant aux exigences du milieu, s'est manifesté dans chaque pays sous différentes formes.

La guerre a activé ce mouvement, mais elle lui a imprimé provisoirement son caractère propre ; elle a imposé la dictature de l'État dans l'intérêt suprême de la défense national ; de plus, les formes d'organisation suscitées par la guerre avaient un objectif immédiat : répartir équitablement les importations collectives effectuées directement par l'État ou avec son concours. Mais la dictature de l'État n'a pas survécu aux circonstances qui l'avaient fait naître. Toutefois, espérons-le, les ententes provoquées d'abord par l'impulsion d'en haut poursuivront leur ouvre et la développeront, par la volonté consciente des intéressés, volonté clairement manifestée par certains d'entre eux.

La politique du Gouvernement a reçu la meilleure des consécrations le jour où nos industriels ont compris qu'ils devaient unir leurs efforts pour transporter la concurrence du 
terrain national, où les victimes ne peuvent nous être indifférentes, sur le terrain international.

Les syndicats industriels de producteur, pour acquérir toute leur vigueur, doivent tirer d'eux-mêmes leur principe vital. Le syndicat obligatoire dont s'accommode la docilité allemande ne s'acclimatera pas facilement en France. Les membres de nos syndicats devront accepter de leur plein gré les disciplines volontaires; leurs facultés d'initiative n'en seront pas amoindries; bien au contraire, l'initiative collective est une forme supérieure de l'initiative privée. Que nos producteurs veuillent bien méditer cette pensée de Bergson: "la liberté est grande source d'énergie, à condition toutefois que les volontés individuelles se règlent méthodiquement sur une fin commune. "S'ils savent se plier aux disciplines nécessaires qui, acceptées et non subies, rendent plus féconds les efforts individuels, ils ne mériteront plus le reproche que Colbert adressait aux négociants de son temps: "Ces Messieurs veulent chacun leur barque, plutôt que de s'associer pour posséder, comme les Hollandais, des grands bateaux». Ce n'est pas au lendemain de la guerre qu'ils oublieront qu'ils naviguent tous sous le pavillon de la France.

De ce que l'État ne peut, à lui seul, donner les instruments d'action aux syndicats constitués en vue d'organiser la production, il serait inadmissible de prétendre qu'il doit les ignorer s'ils existent. Certains ont pu considérer leur existence comme constituant, pour ellemême, un danger : leurs craintes sont exagérées. Le péril serait dans l'irrémédiable faiblesse de nos producteurs condamnés à l'isolement; la force n'est à craindre que lorsqu'on en abuse. Or les abus des groupements de producteurs sont évitables si ces organes, avec des statuts approuvés par les pouvoirs publics, fonctionnent au grand jour de la publicité, comme les sociétés anonymes elles-mêmes dont ils constituent une modalité portée à la suprême puissance. L'État ne participant pas aux risques ne devrait pas intervenir dans la direction, mais, représentant de la collectivité nationale, de l'ensemble des consommateurs, il doit avoir un droit de regard, c'est-à-dire de contrôle, sur le faisceau formidable des forces de production concentrées et organisées.

Les syndicats de producteurs sont des syndicats patronaux, mais les travailleurs sont prêts à collaborer avec eux; ils ne refuseraient, nous en sommes convaincus, leur concours que si les ententes entre producteurs pouvaient contrarier leurs prétentions légitimes à participer aux résultats obtenus par une action commune. Or une forte organisation de la production est la condition essentielle à la fois de l'amélioration immédiate du sort de l'ouvrier et de son accession ultérieure à une situation économique supérieure.

En effet, les syndicats industriels de producteurs - c'est là leur raison d'être- éviteront, par l'organisation de la production, les perturbations économiques; ils empêcheront donc les soubresauts des prix qui, à la longue, déterminent tant de troubles dans les conditions du travail lui-même; le prix de vente n'est pas maintenu par une majoration au détriment du consommateur ; c'est le prix de revient qui est réduit ; il n'y a pas exploitation du salarié ou du consommateur, mais victoire sur la nature et sur la routine.

Encore et surtout, l'organisation du capital stimule et provoque l'organisation des travailleurs. Les promoteurs du mouvement syndicaliste, par une compréhension très juste des choses, ont toujours préféré une société capitaliste vigoureuse à une société capitaliste débile et inorganisée. Lorsque se produira dans toutes les industries la pénétration réciproque des deux forces composantes de la production, on tendra à la réalisation progressive du désir exprimé par Waldeck-Rousseau : "Il faut que le capital travaille et que le travail possède."

Le succès durable de tout programme de suractivité économique est lié à cette collaboration intime du capital et du groupement professionnel, grâce à laquelle l'ouvrier sera intéressé à la production. Que servirait de donner à celui-ci, par l'enseignement technique et l'aménagement scientifique de l'usine, la capacité de produire davantage s'il 
n'avait par la volonté de déployer tout son effort dans les limites de cette capacité accrue? L'intensification doit se produire aux sources mêmes de la production; il en sera ainsi si le travailleur veut, dans la mesure où il peut. L'augmentation méthodique du rendement de la main d'ouvre a donc un corollaire nécessaire : la participation de l'entreprise et de l'ouvrier aux résultats obtenus par un effort commun.

Ainsi, le groupement syndical doit jaillir spontanément des milieux de la production où la concentration industrielle moderne en a déposé les germes; les forces de création et d'organisation qui sont en nous doivent se développer librement; rien de commun avec les rouages d'un mécanisme administratif que l'on crée de toutes pièces et auquel on donne l'impulsion d'en haut. Malheureusement, trop nombreux sont ceux qui restent indifférents aux manifestations les plus convaincantes des avantages de l'effort collectif. Le Ministre du commerce devait s'associer à l'œuvre de ceux qui, conscients du véritable intérêt de la production nationale, ouvrent les voies de l'avenir.

Pour assurer cette collaboration des pouvoirs publics et des forces de production, j'ai dî m'appuyer sur ce qui existe et non sur ce qui sera. Nous possédons en France une institution centenaire qui réunit en assemblées représentatives les intérêts de toute une circonscription, qui, dotée d'un budget, donc de moyens d'action, a su, depuis 20 ans, se moderniser et produire des résultats. J'ai vu dans les Chambres de commerce les points de cristallisation autour desquels s'organiseraient peu à peu les initiatives régionales. Mais la plupart des Chambres de commerce étaient sans moyens d'action, livrées à elles-mêmes. J'ai estimé qu'il convenait tout d'abord de grouper les forces éparses sur le territoire, de les associer dans une action commune et de leur donner, dans les centres qui ont un pouvoir de rayonnement régional, une représentation qui leur permit de devenir d'utiles auxiliaires du pouvoir central dans l'œuvre qu'il poursuit.

Fidèle au principe de la liberté organisée, je n'ai pas voulu imposer aux Chambres de commerce une réforme bureaucratique. Je leur ai exposé les avantages d'une vie régionale plus intense d'où sortirait un accroissement d'activité pour notre production et nos échanges. D'ailleurs, la réforme n'était viable qu'avec leur libre concours, puisque les Chambres de Commerce doivent simplement mettre en pratique une disposition légale restée jusqu'ici presque sans application : user du droit qu'elles tiennent de la loi elle-même de constituer des unions en vue de l'étude des questions d'intérêt commun. Les Chambres de commerce ont magnifiquement répondu à la confiance qui leur était témoignée. Elles ont déclaré qu'elles collaboreraient pleinement à l'œuvre à laquelle elles étaient conviées. Et comme les Chambres de Commerce ont le libre choix des centres auxquels elles doivent s'affilier, les régions économiques, loin d'être des créations artificielles du pouvoir, seront déterminées par les courants véritables de l'activité industrielle et commerciale.

Le cadre de la région économique une fois délimité par voie d'ententes entre le Ministère et les Chambres de Commerce, nous nous sommes efforcés de remplir ce cadre de manière à développer et à organiser les forces productives de la région et nous avons donné une vie permanente, à côté du bureau régional des Chambres de Commerce, au Comité régional d'Action économique, siégeant au chef-lieu de la région, qui, créé par le Ministère de la Guerre, a rendu de grands services au cours des hostilités.

Ce Comité comprend des membres élus et des membres choisis par l'Administration. Une place y est également faite à des délégués des syndicats patronaux et ouvriers. Ces délégués représentent les intérêts d'une corporation déterminée dans le cadre de la région. En effet, les adeptes les plus convaincus du fédéralisme économique n'arrivent à la fédération nationale que par étape et l'intermédiaire de la fédération régionale. Ils reconnaissent que la région, où s'exerce une spécialité professionnelle, peut modifier ses besoins, donner un aspect différent à la solidarité professionnelle et même créer, d'une région à l'autre, des intérêts opposés. Les diversités naturelles sont la cause profonde des besoins économiques différents. 
Sans attendre le plein épanouissement de ces institutions régionales, dont la fédération fera nettement apparaître la nation en ordre de travail, j'ai associé à l'action gouvernementale, pour la rendre plus vivante et moins administrative, pour la renouveler aux sources profondes où s'élabore le progrès, les détenteurs de toutes nos forces de production. Sur mon initiative, les délégués mandatés des chambres syndicales patronales, représentant plusieurs centaines de milliers de chefs d'entreprise industrielles et commerciales, ont constitué 20 groupements ; chacun de ces groupements comprend un certain nombre de syndicats, dont les intérêts sont sinon communs, du moins similaires. Ces conseillers, formés par la pratique quotidienne des affaires, en contact permanent avec les dures réalités, collaboreront efficacement à la restauration économique du pays.

Désormais, par l'intermédiaire de leur Confédération, qui va être constituée sous le régime de la loi de 1884, le Ministre sera directement en rapport avec la masse des industriels et des commerçants, masse non plus amorphe mais organisée. Jusqu'ici le pouvoir central ne connaissait souvent les besoins des industries que lorsque ceux-ci se manifestaient par des crises économiques. Dépourvus des moyens d'investigation et d'information qui lui eussent permis de suivre l'évolution de la maladie, il était incapable de la prévenir et plus encore de la guérir. Désormais, il pourra prévenir et guérir en ayant à sa disposition d'autres moyens d'action que la panacée des articles additionnels à la loi de douane.

Nous sommes maintenant, au cours de l'examen du Rapport, en présence du Ministre du Commerce lui-même, considéré en tant qu'organe de coordination, en tant qu'administration des intérêts supérieurs du Commerce et de l'industrie, sous le contrôle du Parlement souverain. S'il est logiquement constitué, les intérêts particuliers auront le sentiment qu'ils ne peuvent pas se développer et se fortifier au détriment de l'intérêt général, mais en prenant conscience des sacrifices qu'il exige. Le Ministre du Commerce et de l'Industrie devra donc orienter l'activité du pays dans le sens le plus favorable aux intérêts généraux, arbitre légal des conflits inévitables entre branches diverses de la production et du commerce, il devra indiquer aux industriels, aux commerçants, ou plutôt aux groupements qui les représente, le plan général suivant lequel ils doivent diriger leurs efforts pour que l'action des particuliers vienne aider et non entraver celle de l'État. Une nation doit avoir, comme une maison de commerce ou une industrie, un programme, un plan d'action dans l'ordre économique, programme établi avec méthode et réalisé avec persévérance. Ce plan, élaboré avec le concours des groupements qualifiés, sera, à l'intérieur, de favoriser le développement de la production sous toutes ses formes, par les moyens précédemment exposés, à l'extérieur, de provoquer toutes les mesures nécessaire de défense et d'expansion.

L'arme défensive, que nous trouvons dans l'arsenal de l'économie politique traditionnelle, c'est le droit de douane. Il serait prématuré de discuter dans ses détails un régime douanier dont les principes généraux seront conditionnés par le traité de paix. Nous devons cependant ne pas nous laisser surprendre par les événements. Aussi, le Ministère du Commerce a-t-il consacré tout son effort à nous réserver une liberté aussi grande que possible dans l'ordre économique international, de manière à pouvoir prendre toute les mesures que comportera la situation nouvelle. En vue d'obtenir cette complète liberté, le Gouvernement a dénoncé les traités et conventions de commerce conclus avant la guerre. Après avoir, d'accord avec les commissions parlementaires, délibérément abandonné le régime de la clause générale de la nation la plus favorisée que nous avait imposé le traité de Francfort et dont l'application nous fut si funeste, et pour permettre de négocier plus aisément la conclusion des accords destinés à se substituer aux accords dénoncés, j'ai soumis au Parlement un texte qui laissera au Gouvernement la faculté de négocier dans l'intervalle compris entre le tarif général et le tarif minimum. Enfin, pour lutter efficacement contre les tentatives de dumping des États peu scrupuleux, le Ministre du Commerce prépare un projet de constitution d'un Office national des prix. 
Ce sont là des perfectionnements du mécanisme douanier; il sera plus important encore de le pénétrer d'un esprit nouveau. Le droit de douane ne doit plus être une simple prime à la routine et à la paresse, mais un instrument de développement de l'activité nationale. Désormais ne doivent être protégées et encouragées que les industries qui feront effort pour se défendre elles-mêmes, dans la mesure de leurs moyens, contre la concurrence de l'étranger.

D'accord avec le Gouvernement, aidées par lui dans toute la mesure du possible, ces industries devront tout faire pour comprimer les prix de revient, les droits protecteurs devant être destinés à compenser l'écart qui restera incompressible entre les frais de production en France et à l'étranger. Dans l'étude de la mise au point des tarifs, qui ne peut être définitive tant que les prix n'auront pas atteint un certain état d'équilibre, les rapports recueillis à la suite de l'enquête prescrite par M. Lemery, Sous-Secrétaire d'État au Commerce, fourniront les éléments de documentation des plus utiles.

Les droits de douane, révisés et assainis, ne sont pas les seules mesures de protection à envisager. Notre commerce et notre industrie demandaient à être défendus contre l'intrusion non dissimulée et contre l'influence occulte, plus dangereuse encore, de l'activité économique étrangère sur notre territoire. A cet effet, il importait de procurer aux intéressés les moyens de se renseigner exactement et rapidement sur la nationalité des commerçants et des sociétés commerciales établis en France. Tel est l'objet de la loi récemment promulguée, sur le registre du commerce, organisant ce que l'on a appelé justement "l'état civil» des commerçants. Dans le même ordre d'idées, un projet de loi a été déposé en vue de d'instituer, à l'usage des représentants et des voyageurs de commerce français et étrangers exerçant en France leur profession, une carte d'identité professionnelle, qui indiquera la nationalité du commerçant ou du voyageur, tout en renseignant exactement sur les établissements représentés. Une autre série de mesures actuellement à l'étude aura pour but de réglementer l'exercice de certaines professions ou entreprises par des étrangers et de subordonner l'emploi de la qualification de Français à certaines conditions justifiant cette dénomination. Le Ministre du Commerce attache une particulière importance à l'institution des marques collectives et à la répression des fausses indications d'origine. Des projets de loi ayant pour but de garantir la loyauté dans les transactions commerciales ont été déposés. L'un des principaux, celui qui concerne les appellations régionales, est à la veille d'être définitivement adopté par le Parlement.

Lorsque nous aurons, par cet ensemble de réformes, "mis de l'ordre dans la maison", nous serons plus forts pour porter notre action à l'extérieur et plus aptes à y trouver le succès. L'État, ici, a un rôle éminent à jouer que l'on est unanime à lui reconnaître : grands travaux publics, crédits à l'exportation, agents commerciaux; c'est à lui qu'incombe la direction et la concentration énergique des efforts.

Tout d'abord, par les chemins de fer, les fleuves et les canaux, l'État devra ouvrir des accès vers les marchés étrangers. La France, grâce à l'heureux développement de ses côtes, est non seulement, par Marseille, le port de l'Orient, mais encore la façade de l'Europe sur l'Océan Atlantique, cette Méditerranée de la civilisation de demain. Dans cette façade, des fenêtres, plus largement ouvertes qu'aujourd'hui, devront être percées, je veux dire des ports, pourvus de tout l'outillage nécessaire, dans lesquels les navires pourront opérer leurs changements et leurs déchargements sans éprouver des retards préjudiciables. A ces ports devront aboutir les grandes voies ferrées internationales qui y porteront les marchandises de l'Europe centrale et orientale. De ces ports rayonnera dans le monde entier notre marine marchande reconstituée grâce à l'effort de l'armement, aux reprises effectuées sur l'ennemi et à l'exécution du programme de reconstruction soumis au Parlement par M. Buisson, Commissaire aux Transports et à la Marine marchande. Quand nous aurons un nombre de bateaux suffisant pour assurer notre trafic, nous bénéficierons des sommes énormes payées 
chaque année aux armateurs étrangers qui, avant la guerre atteignaient le demi-milliard et, au cours de la guerre, ont représenté plusieurs milliards. Actuellement, cette lourde rançon fait peser une charge écrasante sur notre change, charge qu'il est du devoir primordial du Gouvernement et de l'armement de faire disparaître dans toute la mesure du possible.

Ces moyens de transport accrus ne fonctionneront à plein rendement que sous l'impulsion de nos organes directeurs d'expansion économique. Le Parlement est saisi d'un projet de loi sur la réorganisation de l'Office national du Commerce extérieur, qui deviendra le grand établissement de propagande française à l'étranger. Il disposera d'informations précises, rapidement transmises à nos exportateurs et à nos producteurs, informations recueillis sur tous les points du monde par nos agents commerciaux, sous la direction des attachés commerciaux devenus les chefs de notre représentation commerciale à l'étranger. Des offices commerciaux, dont déjà cinq existent en Suisse, en Angleterre, en Espagne, en Italie et en Russie, offriront à nos industriels et à nos commerçants les avantages d'un compte collectif de vente; plusieurs autres sont en voie de constitution pour l'Orient, avec des agences en Égypte, en Syrie, en Turquie, en Grèce, pour la Hollande et pour la Roumanie. Ils sont organisés de manière à faciliter à nos exportateurs la conclusion de nouvelles affaires, à les aider dans la recherche des débouchés, dans le choix des représentants ; ils renseignent nos commerçants sur les questions de transports, de douanes, de crédit et ils se tiennent à leur disposition pour tout ce qui peut intéresser le développement de leurs affaires; ils préparent des représentations temporaires de modèles et d'échantillons.

Enfin, c'est un lieu commun de dire que la principale cause de l'insuffisance de nos exportations a été l'infériorité de notre outillage bancaire; la création d'une banque d'exportation, ouvrant à ses clients des crédits à long terme, est l'instrument indispensable de notre succès. Nous avons pu grouper les représentants de l'industrie, du commerce et de la banque dans ce but et, dès maintenant, on peut tenir l'établissement, dont notre commerce d'exportation a si grand besoin, comme constitué.

Telles sont, selon les conclusions du rapport que j'ai l'honneur de vous présenter, les conditions générales qui doivent présider à la réorganisation économique de la France. Elles exigeront, de la part de l'État, un effort financier plus grand que dans le passé. De tous les départements ministériels, le Ministre du Commerce est celui dont les dépenses d'administration centrale sont les plus faibles. A la veille de la guerre, elles s'élevaient annuellement à 439.000 fr., alors que le Service des Beaux-Arts absorbait $487.000 \mathrm{fr}$. République athienne, la France faisait autant de sacrifices pour ce qui peut contribuer à la beauté de la vie que pour ce qui était nécessaire au développement de sa vie industrielle. Un projet de loi réorganisant le Ministère du Commerce et le mettant à même de remplir le rôle que les circonstances lui imposent sera déposé dans quelques jours sur le Bureau de la Chambre.

Les événements qui se déroulent dans le monde prouvent que les plus brillantes civilisations doivent s'appuyer sur les solides réalités économiques. Ce sont ces réalités qui forment l'objet des travaux du Comité consultatif des arts et manufactures et de la Direction des Etudes techniques du Ministère du Commerce. Les trois volumes du Rapport général, en même temps qu'une encyclopédie de l'industrie contemporaine, forment la base des études qui vont se poursuivre en collaboration avec les groupements industriels patronaux et ouvriers et qui conduiront rapidement à l'adoption des solutions qui s'imposent pour la prompte restauration du pays.

Nos industriels, au cours d'une crise économique sans précédent, ont donné la mesure de leur magnifique activité ; nos ouvriers ont démontré que leur labeur pouvait puissamment féconder les capitaux mis à la disposition du travail. Aux uns et aux autres, l'État doit plus que la seule liberté : elle ressemblerait trop à une abdication et à une défaillance. Il saura 
leur procurer les moyens d'action collectifs, l'outillage matériel et les organes d'expansion. Dans le monde entier, où jamais notre drapeau n'a brillé d'un tel éclat, où nos rivaux nous précèdent avec une hâte fébrile, on attend impatiemment des manifestations dignes de notre réputation ancienne et de notre prestige nouveau. Le temps presse, si nous voulons éviter les sévérités de l'Histoire. Mais, j'en ai la conviction, la France qui a su vaincre, sous votre énergique impulsion, saura triompher de toutes les difficultés grâce à son travail, à son ingéniosité, à son esprit d'initiative, à sa ténacité ; elle étonnera le monde par la rapidité de sa restauration industrielle et par l'ampleur de son expansion économique.

Veuillez agréer, Monsieur le Président, l’hommage de mon respectueux dévouement.

29 mars 1919.

CLÉMENTEL. 